\title{
Semiclassical many-mode Floquet theory. III. SU(3) dynamical evolution of three-level systems in intense bichromatic fields
}

\author{
Tak-San Ho and Shih-I Chu \\ Department of Chemistry, University of Kansas, Lawrence, Kansas 66045
}

(Received 30 August 1984)

\begin{abstract}
The SU(3) dynamical evolution of three-level systems at two-photon resonance induced by two strong linearly polarized monochromatic fields is studied exactly by means of the semiclassical many-mode Floquet theory (MMFT) recently developed by the authors. Within the rotating-wave approximation (RWA), Hioe and Eberly have recently shown that the eight-dimensional SU(3) coherent vector $\mathbf{S}$ characterizing the time evolution of three-level systems can be factored into three independent vectors of dimensions three, four, and one, at appropriate two-photon resonance conditions. In practice, however, if the laser-atom interactions occur away from the two-photon resonance, or if the RWA is not valid, etc., this Gell-Mann-type SU(3) dynamical symmetry will be broken. It is shown in this paper that instead of solving the time-dependent generalized Bloch equations, the SU(3) dynamical evolution of the coherent vector $\mathbf{S}$ as well as various symmetry-breaking effects can be expediently studied by the use of the MMFT and expressed in terms of a few timeindependent quasienergy eigenvalues and eigenvectors. Furthermore, we have extended the generalized Van Vleck (GVV) nearly degenerate perturbation theory to an analytical treatment of the twomode Floquet Hamiltonian. This reduces the infinite-dimensional time-independent Floquet Hamiltonian into a $3 \times 3$ effective Hamiltonian, from which useful analytical properties of the SU(3) coherent vector can be easily obtained. The combination of the MMFT and the GVV method thus greatly facilitates the study of the dynamical evolution. Pictorial comparison of the exact and the RWA results of the time evolution of the eight-dimensional coherent vector under several different physical conditions is presented and discussed at length.
\end{abstract}

\section{INTRODUCTION}

The dynamical evolution of three-level systems in the presence of two strong monochromatic fields has been the subject of fundamental significance to a wealth of important problems. Examples of current interests are twophoton NMR on spin-1 systems, ${ }^{1}$ double-resonance spectroscopy of atoms and molecules, ${ }^{2}$ two-photon coherence, ${ }^{3,4}$ population trapping, ${ }^{5}$ resonance Raman scattering, ${ }^{6}$ and many others.

It has long been known that for two-level systems the description of magnetic and optical resonance phenomena can be greatly simplified by the use of the Bloch spin or pseudospin vector. ${ }^{7,8}$ However, extension of the vector description to more complex systems has been achieved only recently. Hioe and Eberly ${ }^{9}$ found that the dynamical evolution of $N(\geq 3)$-level systems can be expressed in terms of the generalized rotation of an $\left(N^{2}-1\right)$ dimensional real coherence vector whose property can be analyzed by appealing to the $\mathrm{SU}(N)$ group symmetry. In particular, the time evolution of three-level systems can be described by a classical coherent vector of constant length rotating in an eight-dimensional space. ${ }^{9,10}$ Unlike the conventional two-photon vector model $^{3}$ which can only apply to limited cases, the SU(3) vector model is exact and is thus free from any restriction. Earlier Zur and Pines ${ }^{11}$ also proposed a formalism based upon the fictitious spin$\frac{1}{2}$ operators, also a set of the group $\operatorname{SU}(N)$ generators, to describe the time evolution of spin systems with $I>\frac{1}{2}$, but they did not exploit the geometric notion of the rotating coherence vector. Zur and $\mathrm{Vega}^{1}$ in a recent paper have studied, theoretically and experimentally, the detailed dynamics of two-photon ${ }^{2} \mathrm{D}$ NMR on a deuterated malonic acid irradiated by pulsed double radiofrequency (rf) fields in terms of fictitious spin- $\frac{1}{2}$ operators.

There are, in general, three types, i.e., cascade, " $V$," and lambda, of three-level systems which are most widely studied. All these three types assume opposite parity for adjacent levels, i.e., level 2 is of different parity from levels 1 and 3 which are, in turn, of the same parity. In this paper we shall consider only the cascade type of the three-level system as an example, but the approach to be developed in the following can be readily extended to other types. Most theoretical studies on these three-level systems in the presence of two nearly resonant radiation fields have been confined to the so-called rotating-wave approximation (RWA) that assumes one radiation field drives only one electric-dipole allowed transition. While it is true that RWA serves a coarse approximation in many circumstances, many nonlinear higher-order effects caused by non-RWA terms can unveil important and interesting subtleties of the dynamics of the system under study. To explore the influence of non-RWA factors we further distinguish two physically different cascade three-levels, one with large disparity in allowed transition frequencies, see Fig. 1(a), and the other with small disparity, see Fig. 1(b). With the assumption that one field drives only one transition and within the rotating-wave 


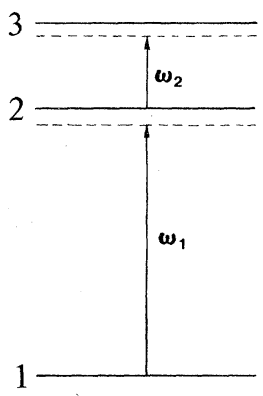

(a)

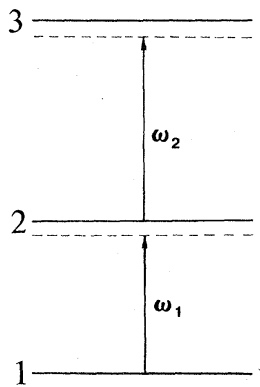

(b)
FIG. 1. Two types of cascade three-level systems having nonvanishing dipole matrix elements between levels 1 and 2 , and 2 and 3. (a) Type $I$ has large disparity between the two transition frequencies, i.e., $\left(E_{2}-E_{1}\right) \gg\left(E_{3}-E_{2}\right)$, and (b) type II has small disparity between the two transition frequencies, i.e., $\left(E_{2}-E_{1}\right) \approx\left(E_{3}-E_{2}\right)$, but $\left(E_{2}-E_{1}\right) \neq\left(E_{3}-E_{2}\right)$.

approximation, Hioe and Eberly, in a series of papers, ${ }^{12}$ have shown that the three-level eight-dimensional coherent vector can be further factored into three independent vectors of dimensions three, four, and one, and analytic expressions of the coherent vector can be derived under certain conditions. They have also investigated the problem of the adiabatic following in the three-level system explicitly. ${ }^{13}$

Much of recent multiphoton research is couched in terms of semiclassical theory, particularly the Floquet theoretical approaches. The Floquet formalism has provided practical and powerful nonperturbative methods for studying multiphoton excitation, ${ }^{14}$ ionization, ${ }^{15}$ and dissociation ${ }^{16}$ processes. The conventional Floquet approaches, however, require the Hamiltonians to be explicitly periodic in time and are therefore applicable only to problems involving single monochromatic radiation field. In two previous papers, ${ }^{17,18}$ a generalized many-mode Floquet theory (MMFT) has been developed, making possible for the first time exact reformulation of the time-dependent problem of any $N$-level system (with finite $N$ ) exposed to polychromatic fields as an equivalent time-independent infinite-dimensional eigenvalue problem. In paper $I,{ }^{17}$ we have presented the general formulation of the MMFT which is an exact extension of Shirley's single-mode Floquet theory. ${ }^{19}$ In paper II, ${ }^{18}$ we have fully explored the detailed nonlinear response of two-level systems under the influence of intense bichromatic fields, and we have derived useful analytical expressions for multiphoton transition probabilities, resonance shifts and widths, etc., using Salwen's nearly degenerate perturbation theory. ${ }^{20}$

In this paper we shall extend the many-mode Floquet theory to the problem of the dynamical evolution of three-level systems at two-photon resonance illuminated by two strong, linearly polarized monochromatic radiation fields. In addition to the numerically exact results, we shall make use of the generalized Van Vleck (GVV) degenerate perturbation theory ${ }^{21,22}$ to obtain the analytical expressions for the wave function, to the first order in Rabi frequencies, and for the quasienergies, to the third order. The time evolution of the three-level coherence vector is presented along with the time-dependent population distributions over various levels. The study of the SU(3) dynamical evolution as well as various symmetrybreaking effects (due to the breakdown of the RWA, or two-photon-off resonance conditions, etc.) is greatly facilitated by the use of the MMFT-GVV method. The two types, i.e., Figs. 1(a) and 1(b), of the three-level system aforementioned shall serve as examples to demonstrate the utility of our approach and to illustrate the salient features of three-level systems interacting simultaneously with two strong fields.

In Sec. II we present a brief account of the many-mode Floquet theory. The generalized Van Vleck degenerate perturbation treatment of the (time-independent) manymode Floquet Hamiltonian is discussed in Sec. III. The analytical properties of the SU(3) coherent vector is analyzed in Sec. IV. Detailed pictorial comparison of the exact and RWA results of the dynamical evolution of the eight-dimensional coherent vector is presented and discussed in Sec. V. This is followed by a conclusion in Sec. VI. Atomic units are used throughout unless otherwise specified.

\section{SEMICLASSICAL MANY-MODE FLOQUET THEORY}

The time evolution of an arbitrary $N$-level system interacting with two linearly polarized monochromatic electromagnetic fields is governed, within the electric-dipole approximation, by the time-dependent Schrödinger equation

$$
i \frac{d}{d t} \Psi(t)=\hat{H}(t) \Psi(t)
$$

where

$$
\hat{H}(t)=\hat{H}_{0}+\sum_{i=1}^{2} \hat{V}_{i}(t) .
$$

Here $\hat{H}_{0}$ is the unperturbed Hamiltonian of the $N$-level system with eigenenergies $\left\{E_{\alpha}\right\}$ and eigenfunctions $\left\{\psi_{\alpha}\right\}$, i.e.,

$$
\widehat{H}_{0} \psi_{\alpha}=E_{\alpha} \psi_{\alpha}, \quad \alpha=1,2, \ldots, N \text {. }
$$

The interaction Hamiltonian $\widehat{V}_{i}(t)$ is given by

$$
\widehat{V}_{i}(t)=-\mu \cdot \epsilon_{i} \cos \left(\omega_{i} t+\theta_{i}\right), \quad i=1,2
$$

where $\boldsymbol{\mu}$ is the electric-dipole moment operator, and $\boldsymbol{\epsilon}_{i}$, $\omega_{i}$, and $\theta_{i}$ specify the amplitude, frequency, and initial phase, respectively, of the $i$ th classical field. It has been shown, in papers $I^{17}$ and $I I,{ }^{18}$ that the time-evolution operator $\hat{U}\left(t ; t_{0}\right)$, defined in the basis of the unperturbed wave functions $\left\{\psi_{\alpha}\right\}$ of Eq. (2) and satisfying the initial condition $\hat{U}\left(t_{0} ; t_{0}\right)=\underline{I}$ with $t_{0}$ the initial time and $\underline{\hat{I}}$ an $N \times N$ identity matrix, can be written as

$$
\underline{\hat{U}}\left(t ; t_{0}\right)=\underline{\hat{F}}(t)\left[\underline{\hat{F}}\left(t_{0}\right)\right]^{\dagger} \text {. }
$$

Here $\hat{F}(t)$ is a unitary operator and can be expanded in terms of a double Fourier series, namely, in matrix form, 


$$
F_{\beta \alpha}(t)=\exp \left(-i \lambda_{\alpha m_{1} m_{2}} t\right) \sum_{n_{1}=-\infty}^{\infty} \sum_{n_{2}=-\infty}^{\infty}\left\langle\beta, n_{1}, n_{2} \mid \lambda_{\alpha m_{1} m_{2}}\right\rangle \exp \left[i\left(n_{1} \omega_{1}+n_{2} \omega_{2}\right) t\right]
$$

with $\left\{\lambda_{\alpha m_{1} m_{2}}\right\}$ and $\left\{\left\langle\beta, n_{1}, n_{2} \mid \lambda_{\alpha m_{1} m_{2}}\right\rangle\right\}$ being the bichromatic quasienergy eigenvalues and the components of eigenvectors of the time-independent infinite-dimensional eigenvalue equation

$$
\sum_{\gamma=1}^{N} \sum_{k_{1}=-\infty}^{\infty} \sum_{k_{2}=-\infty}^{\infty}\left\langle\beta, n_{1}, n_{2}\left|\hat{H}_{F}\right| \gamma, k_{1}, k_{2}\right\rangle\left\langle\gamma, k_{1}, k_{2} \mid \lambda_{\alpha m_{1} m_{2}}\right\rangle=\lambda_{\alpha m_{1} m_{2}}\left\langle\beta, n_{1}, n_{2} \mid \lambda_{\alpha m_{1} m_{2}}\right\rangle
$$

Here greek letters $\alpha, \beta, \gamma, \ldots$ denote the unperturbed $N$ levels defined in Eq. (2), roman letters $k, m, n, \ldots$ are Fourier indices, and the two-mode Floquet Hamiltonian $\hat{H}_{F}$ is defined in the generalized Floquet-state basis $\left\{\left|\alpha, m_{1}, m_{2}\right\rangle \equiv|\alpha\rangle \otimes\left|m_{1}\right\rangle \otimes\left|m_{2}\right\rangle\right\}:$

$$
\begin{aligned}
& \left\langle\beta, n_{1}, n_{2}\left|\hat{H}_{F}\right| \gamma, k_{1}, k_{2}\right\rangle \\
& =H_{\beta \gamma}^{n_{1}-k_{1}, n_{2}-k_{2}}+\left(n_{1} \omega_{1}+n_{2} \omega_{2}\right) \delta_{\beta \gamma} \delta_{n_{1} k_{1}} \delta_{n_{2} k_{2}},
\end{aligned}
$$

with

$H_{\beta \gamma}^{n_{1} n_{2}}=E_{\beta} \delta_{\beta \gamma} \delta_{n_{1} 0} \delta_{n_{2} 0}+\sum_{i=1}^{2} V_{\beta \gamma}^{(i)}\left(\delta_{n_{i} 1}+\delta_{n_{i},-1}\right) \delta_{n 0}$

and

$$
V_{\beta \gamma}^{(i)}=-\frac{1}{2}\left\langle\beta\left|\mu \cdot \epsilon_{i}\right| \gamma\right\rangle \cdot e^{i \theta_{i} n_{i}},
$$

where $n=n_{2}$ if $i=1$, and $n=n_{1}$ if $i=2$.
We remark, in particular, that the matrix elements $\left\{F_{\beta \alpha}\right\}$ of the unitary operator $\widehat{F}(t)$ are independent of Fourier indices $m_{1}$ and $m_{2}$ appearing explicitly in Eq. (5) because of the periodic relations, embodied in the structure of $\hat{H}_{F}$ defined in Eq. (7),

$$
\lambda_{\alpha, m_{1}+k_{1}, m_{2}+k_{2}}=\lambda_{\alpha m_{1} m_{2}}+k_{1} \omega_{1}+k_{2} \omega_{2}
$$

and

$$
\begin{aligned}
& \left\langle\beta, n_{1}+k_{1}, n_{2}+k_{2} \mid \lambda_{\alpha, m_{1}+k_{1}, m_{2}+k_{2}}\right\rangle \\
& =\left\langle\beta, n_{1}, n_{2} \mid \lambda_{\alpha m_{1} m_{2}}\right\rangle .
\end{aligned}
$$

By employing Eq. (4) via (9), various versions of transition probabilities of the system going from an initial state $|\alpha\rangle=\psi_{\alpha}$ to an arbitrary final state $|\beta\rangle \equiv \psi_{\beta}$ can be computed easily. ${ }^{17,18}$ In the following we shall be especially interested in (i) the state-to-state time-dependent transition probability

$$
\begin{aligned}
P_{\alpha \beta}\left(t ; t_{0}\right)= & \left|\hat{U}_{\beta \alpha}\left(t ; t_{0}\right)\right|^{2} \\
= & \sum_{m_{1}, m_{2}} \sum_{k_{1}, k_{2}}\left\langle\beta, k_{1}, k_{2}\left|\exp \left[-i \hat{H}_{F}\left(t-t_{0}\right)\right]\right| \alpha, 0,0\right\rangle \\
& \quad \times \exp \left[i\left(m_{1} \omega_{1}+m_{2} \omega_{2}\right) t_{0}\right]\left\langle\alpha, m_{1}, m_{2}\left|\exp \left[i \hat{H}_{F}\left(t-t_{0}\right)\right]\right| \beta, k_{1}, k_{2}\right\rangle,
\end{aligned}
$$

where $t$ and $t_{0}$ are, respectively, the final and initial times, and (ii) the long time-averaged, i.e., averaged over the random initial times $t_{0}$ and all possible elapsed time intervals $\left(t-t_{0}\right)$, transition probability

$$
\overline{\boldsymbol{P}}_{\alpha \beta}=\sum_{k_{1}, k_{2}} \sum_{\gamma, l_{1}, l_{2}}\left|\left\langle\beta, k_{1}, k_{2} \mid \lambda_{\gamma l_{1} l_{2}}\right\rangle\left\langle\lambda_{\gamma l_{1} l_{2}} \mid \alpha, 0,0\right\rangle\right|^{2} .
$$

\section{GENERALIZED VAN VLECK (GVV) DEGENERATE PERTURBATION TREATMENT OF TWO-MODE FLOQUET HAMILTONIAN}

We shall consider only two types of three-level systems shown in Figs. 1(a) and 1(b). Furthermore we assume that (1) detunings $\Delta_{1} \equiv\left(E_{2}-E_{1}\right)-\omega_{1}<<\left(E_{2}-E_{1}\right)$ and $\Delta_{2}$ $\equiv\left(E_{3}-E_{2}\right)-\omega_{2}<<\left(E_{3}-E_{2}\right)$, and (2) Rabi frequencies $\Omega_{i} \equiv \frac{1}{2}\left\langle 1\left|\mu \cdot \epsilon_{i}\right| 2\right\rangle$ and $\Omega_{i}^{\prime} \equiv \frac{1}{2}\left\langle 2\left|\mu \cdot \epsilon_{i}\right| 3\right\rangle$, with $i=1,2$, are small in magnitude compared with detunings $\Delta_{1}$ and $\Delta_{2}$, or the quantity $\left|\left(E_{2}-E_{1}\right)-\left(E_{3}-E_{2}\right)\right|$. We are thus only interested in the resonant, or nearly resonant, twophoton processes of cascade three-level systems in the presence of two strong fields.

Under the two conditions assumed in the previous paragraph, the diagonal elements $\left\langle 1, m_{1}, m_{2}\left|\hat{H}_{F}\right| 1, m_{1}, m_{2}\right\rangle$, $\left\langle 2, m_{1}-1, m_{2}\left|\hat{H}_{F}\right| 2, m_{1}-1, m_{2}\right\rangle, \quad$ and $\left\langle 3, m_{1}-1\right.$, $\left.m_{2}-1\left|\hat{H}_{F}\right| 3, m_{1}-1, m_{2}-1\right\rangle$, with $m_{1}$ and $m_{2}$ arbitrary integers, of the multimode Floquet Hamiltonian $\hat{H}_{F}$ form a nearly degenerate trio. The perturbed eigenvalues and eigenvectors with which the three nearly degenerate diagonal elements are associated can then be evaluated approximately and simultaneously by some sort of nearly degenerate perturbation method. Among the existing perturbation approaches, we shall adopt a version developed firstly by Van Vleck, ${ }^{23}$ generalized by Kirtman, ${ }^{21}$ further exploited by Certain and Hirschfelder, ${ }^{22}$ and recently used by Aravind and Hirschfelder in two-level studies. ${ }^{24}$

The general idea behind this technique, hereafter called the generalized Van Vleck (GVV) theory, is to block diagonalize the time-independent Hamiltonian [such as $\hat{H}_{F}$ in Eq. (7)], so that the coupling between the model space and the remainder of the configuration space (called the external space) diminishes to a desired order. The perturbed eigenvalues and eigenvectors corresponding to the set of 
nearly degenerate states chosen can thus be solved approximately by considering the model-space Hamiltonian as an effective total Hamiltonian of the system. In the following we shall illustrate the GVV approach in the twophoton processes of three-level systems depicted in Figs. 1(a) and 1(b); many salient features of the GVV technique shall then become clear.

We shall concentrate on three nearly degenerate states $|1,0,0\rangle,|2,-1,0\rangle$, and $|3,-1,-1\rangle$ in the derivations below; periodicities (9) and (10) will generate all necessary results. At first we propose that the perturbed modelspace wave functions be written as

$$
\begin{aligned}
& \left|\phi_{100}\right\rangle=\left|\phi_{100}^{(0)}\right\rangle+\left|\phi_{100}^{(1)}\right\rangle+\left|\phi_{100}^{(2)}\right\rangle+\cdots, \\
& \left|\phi_{2,-1,0}\right\rangle=\left|\phi_{2,-1,0}^{(0)}\right\rangle+\left|\phi_{2,-1,0}^{(1)}\right\rangle+\left|\phi_{2,-1,0}^{(2)}\right\rangle+\cdots,
\end{aligned}
$$

and

$$
\begin{aligned}
\left|\phi_{3,-1,-1}\right\rangle= & \left|\phi_{3,-1,-1}^{(0)}\right\rangle+\left|\phi_{3,-1,-1}^{(1)}\right\rangle \\
& +\left|\phi_{3,-1,-1}^{(2)}\right\rangle+\cdots,
\end{aligned}
$$

with

$$
\begin{aligned}
& \left|\phi_{100}^{(0)}\right\rangle=|1,0,0\rangle, \quad\left|\phi_{2,-1,0}^{(0)}\right\rangle=|2,-1,0\rangle, \\
& \left|\phi_{3,-1,-1}^{(0)}\right\rangle=|3,-1,-1\rangle .
\end{aligned}
$$

Correspondingly, the external wave functions, which span the external space, are expressed as

$$
\left|\chi_{p}\right\rangle=\left|\chi_{p}^{(0)}\right\rangle+\left|\chi_{p}^{(1)}\right\rangle+\left|\chi_{p}^{(2)}\right\rangle+\cdots,
$$

where $\left|\chi_{p}^{(0)}\right\rangle$ 's are unperturbed Floquet states other than $|1,0,0\rangle,|2,-1,0\rangle$, and $|3,-1,-1\rangle$. According to the
GVV theory, in first order, every $\left|\chi_{p}\right\rangle$ will be orthogonal to each $\left|\phi_{\alpha m_{1} m_{2}}\right\rangle$, where $\left(\alpha, m_{1}, m_{2}\right)$ can be $(1,0,0)$, $(2,-1,0)$, or $(3,-1,-1)$, provided that

$$
\left\langle\chi_{p}^{(1)} \mid \phi_{\alpha m_{1} m_{2}}^{(0)}\right\rangle+\left\langle\chi_{p}^{(0)} \mid \phi_{\alpha m_{1} m_{2}}^{(1)}\right\rangle=0
$$

for all $p$ and $\left(\alpha, m_{1}, m_{2}\right)$, or

$$
\left|\chi_{p}^{(1)}\right\rangle=-\sum_{\alpha, m_{1}, m_{2}}\left|\phi_{\alpha m_{1} m_{2}}^{(0)}\right\rangle\left\langle\chi_{p}^{(0)} \mid \phi_{\alpha m_{1} m_{2}}^{(1)}\right\rangle \text {. }
$$

If we further require that the perturbed set $\left\{\left|\phi_{\alpha m_{1} m_{2}}\right\rangle\right\}$ be orthonormal, the first-order perturbation function can be written as

$$
\left|\phi_{\alpha m_{1} m_{2}}^{(1)}\right\rangle=\hat{R}_{\alpha m_{1} m_{2}}^{(0)} \hat{H}_{F 1}\left|\phi_{\alpha m_{1} m_{2}}^{(0)}\right\rangle,
$$

where the resolvant operator is defined as

$$
\hat{R}_{\alpha m_{1} m^{2}}^{(0)}=\sum_{p}\left|\chi_{p}^{(0)}\right\rangle\left(E_{\alpha m_{1} m_{2}}^{(0)}-E_{p}^{(0)}\right)^{-1}\left\langle\chi_{p}^{(0)}\right|,
$$

and the perturbation Hamiltonian $\hat{H}_{F 1}$ is the off-diagonal part of the multimode Floquet Hamiltonian $\hat{H}_{F}$ of Eq. (7), i.e., $\hat{H}_{F 1} \equiv \hat{H}_{F}-\hat{H}_{F 0}$ with $\hat{H}_{F 0}$ the diagonal part of $\widehat{H}_{F}$ and having diagonal elements $E_{\alpha m_{1} m_{2}}^{(0)} \equiv E_{\alpha}+m_{1} \omega_{1}$ $+m_{2} \omega_{2}$. Similarly we can also carry out higher-order corrections by imposing the same full orthonormal conditions, but to a higher degree. In our case here we shall be content with the first-order perturbation. By introducing the notations

$$
b_{i}^{(\alpha \beta)} \equiv-\frac{1}{2}\left\langle\alpha\left|\mu \cdot \epsilon_{i}\right| \beta\right\rangle e^{i \theta_{i}}, \quad i=1,2
$$

the first-order model-space perturbation functions can be written explicitly as

$$
\begin{aligned}
\left|\phi_{100}^{(1)}\right\rangle= & \frac{b_{2}^{(12)}}{E_{100}^{(0)}-E_{201}^{(0)}}|2,0,1\rangle+\frac{b_{1}^{(12)}}{E_{100}^{(0)}-E_{211}^{(0)}}|2,1,0\rangle+\frac{\left(b_{2}^{(12)}\right)^{*}}{E_{100}^{(0)}-E_{2,0,-1}^{(0)}}|2,0,-1\rangle, \\
\left|\phi_{2,-1,0}^{(1)}\right\rangle= & \frac{b_{2}^{(12)}}{E_{2,-1,0}^{(0)}-E_{1,-1,1}^{(0)}}|1,-1,1\rangle+\frac{\left(b_{1}^{(12)}\right)^{*}}{E_{2,-1,0}^{(0)}-E_{1,-2,0}^{(0)}}|1,-2,0\rangle+\frac{\left(b_{2}^{(12)}\right)^{*}}{E_{2,-1,0}^{(0)}-E_{1,-1,-1}^{(0)}}|1,-1,-1\rangle \\
& \quad+\frac{b_{2}^{(23)}}{E_{2,-1,0}^{(0)}-E_{3,-1,1}^{(0)}}|3,-1,1\rangle+\frac{b_{1}^{(23)}}{E_{2,-1,0}^{(0)}-E_{300}^{(0)}}|300\rangle+\frac{\left(b_{1}^{(23)}\right)^{*}}{E_{2,-1,0}^{(0)}-E_{3,-2,0}^{(0)}}|3,-2,0\rangle,
\end{aligned}
$$

and

$$
\left|\phi_{3,-1,-1}^{(1)}\right\rangle=\frac{b_{1}^{(23)}}{E_{3,-1,-1}^{(0)}-E_{2,0,-1}^{(0)}}|2,0,-1\rangle+\frac{\left(b_{1}^{(23)}\right)^{*}}{E_{3,-1,-1}^{(0)}-E_{2,-2,-1}^{(0)}}|2,-2,-1\rangle+\frac{\left(b_{2}^{(23)}\right)^{*}}{E_{3,-1,-1}^{(0)}-E_{2,-1,-2}^{(0)}}|2,-1,-2\rangle .
$$

One important feature of the GVV approach is that if the wave functions, e.g., Eqs. (12) and (13), are exact to the $n$th order, the corresponding Hamiltonian matrix on the model space can be computed to the $(2 n+1)$ th order. ${ }^{22}$ To the first order, the model-space Hamiltonian matrix can this be expressed analytically as

$$
\underline{\hat{H}}_{3} \equiv \underline{\hat{H}}_{3}^{(0)}+\underline{\hat{H}}_{3}^{(1)}+\underline{\hat{H}}_{3}^{(2)}+\underline{\hat{H}}_{3}^{(3)},
$$

where

$\left(\underline{\hat{H}}_{3}^{(0)}\right)_{(1,0,0),(1,0,0)}=E_{100}^{(0)}$,

$\left(\underline{\hat{H}}_{3}^{(0)}\right)_{(2,-1,0),(2,-1,0)}=E_{2,-1,0}^{(0)}$,

$\left(\underline{\hat{H}}_{3}^{(0)}\right)_{(3,-1,-1),(3,-1,-1)}=E_{3,-1,-1}^{(0)}$, 
$\left(\underline{\hat{H}}_{3}^{(1)}\right)_{(1,0,0),(2,-1,0)}=\left(\hat{H}_{3}^{(1)}\right)_{(2,-1,0),(1,0,0)}^{*}=b_{1}^{(12)}$,

$\left(\underline{\hat{H}}_{3}^{(1)}\right)_{(2,-1,0),(3,-1,-1)}=\left(\widehat{H}_{3}^{(1)}\right)_{(3,-1,-1),(2,-1,0)}^{*}=b_{2}^{(23)}$,

$\left(\underline{\hat{H}}_{3}^{(2)}\right)_{(1,0,0),(1,0,0)}=\frac{\left|b_{2}^{(12)}\right|^{2}}{E_{100}^{(0)}-E_{201}^{(0)}}+\frac{\left|b_{1}^{(12)}\right|^{2}}{E_{100}^{(0)}-E_{210}^{(0)}}+\frac{\left|b_{2}^{(12)}\right|^{2}}{E_{100}^{(0)}-E_{2,0,-1}^{(0)}}$,

$\left(\underline{\hat{H}}_{3}^{(2)}\right)_{(2,-1,0),(2,-1,0)}=\frac{\left|b_{2}^{(12)}\right|^{2}}{E_{2,-1,0}^{(0)}-E_{1,-1,1}^{(0)}}+\frac{\left|b_{1}^{(12)}\right|^{2}}{E_{2,-1,0}^{(0)}-E_{1,-2,0}^{(0)}}+\frac{\left|b_{2}^{(12)}\right|^{2}}{E_{2,-1,0}^{(0)}-E_{1,-1,-1}^{(0)}}$

$$
+\frac{\left|b_{2}^{(23)}\right|^{2}}{E_{2,-1,0}^{(0)}-E_{3,-1,1}^{(0)}}+\frac{\left|b_{1}^{(23)}\right|^{2}}{E_{2,-1,0}^{(0)}-E_{300}^{(0)}}+\frac{\left|b_{1}^{(23)}\right|^{2}}{E_{2,-1,0}^{(0)}-E_{3,-2,0}^{(0)}},
$$

$\left(\underline{\hat{H}}_{3}^{(2)}\right)_{(3,-1,-1),(3,-1,-1)}=\frac{\left|b_{1}^{(23)}\right|^{2}}{E_{3,-1,-1}^{(0)}-E_{2,0,-1}^{(0)}}+\frac{\left|b_{1}^{(23)}\right|^{2}}{E_{3,-1,-1}^{(0)}-E_{2,-2,-1}^{(0)}}+\frac{\left|b_{2}^{(23)}\right|^{2}}{E_{3,-1,-1}^{(0)}-E_{2,-1,-2}^{(0)}}$,

$\left(\underline{\hat{H}}_{3}^{(2)}\right)_{(1,0,0),(3,-1,-1)}=\left(\hat{H}_{3}^{(2)}\right)_{(3,-1,-1),(1,0,0)}^{*}$

$$
=\frac{1}{2}\left[\frac{b_{1}^{(23)} b_{2}^{(12)}}{E_{3,-1,-1}^{(0)}-E_{2,0,-1}^{(0)}}+\frac{b_{2}^{(12)} b_{1}^{(23)}}{E_{100}^{(0)}-E_{2,0,-1}^{(0)}}\right],
$$

$\left(\underline{\hat{H}}_{3}^{(3)}\right)_{(1,0,0)(2,-1,0)}=\left(\underline{\hat{H}}_{3}^{(3)}\right)_{(2,-1,0),(1,0,0)}^{*}$

$$
\begin{aligned}
= & \frac{\left(b_{2}^{(12)}\right)^{*} b_{2}^{(12)} b_{1}^{(12)}}{\left(E_{100}^{(0)}-E_{201}^{(0)}\right)\left(E_{2,-1,0}^{(0)}-E_{1,-1,1}^{(0)}\right)}+\frac{\left(b_{2}^{(12)}\right)^{*} b_{2}^{(23)} b_{1}^{(23)}}{\left(E_{100}^{(0)}-E_{201}^{(0)}\right)\left(E_{2,-1,0}^{(0)}-E_{3,-1,1}^{(0)}\right)} \\
& +\frac{\left(b_{1}^{(12)}\right)^{*} b_{1}^{(23)} b_{1}^{(23)}}{\left(E_{100}^{(0)}-E_{210}^{(0)}\right)\left(E_{2,-1,0}^{(0)}-E_{300}^{(0)}\right)}+\frac{b_{2}^{(12)} b_{1}^{(23)}\left(b_{2}^{(23)}\right)^{*}}{\left(E_{100}^{(0)}-E_{2,0,-1}^{(0)}\right)\left(E_{2,-1,0}^{(0)}-E_{300}^{(0)}\right)} \\
& +\frac{b_{2}^{(12)}\left(b_{2}^{(12)}\right)^{*} b_{1}^{(12)}}{\left(E_{100}^{(0)}-E_{2,0,-1}^{(0)}\right)\left(E_{2,-1,0}^{(0)}-E_{1,-1,-1}^{(0)}\right)}+\frac{\left(b_{2}^{(12)}\right)^{*} b_{1}^{(23)} b_{2}^{(23)}}{\left(E_{100}^{(0)}-E_{201}^{(0)}\right)\left(E_{2,-1,0}^{(0)}-E_{300}^{(0)}\right)} \\
& -\frac{1}{2} \frac{b_{2}^{(12)} b_{1}^{(23)}\left(b_{2}^{(23)}\right)^{*}}{\left(E_{100}^{(0)}-E_{2,0,-1}^{(0)}\right)\left(E_{3,-1,-1}^{(0)}-E_{2,0,-1}^{(0)}\right)}-\frac{1}{2} b_{1}^{(12)}\left(\left\langle\phi_{100}^{(1)} \mid \phi_{100}^{(1)}\right\rangle+\left\langle\phi_{2,-1,0}^{(1)} \mid \phi_{2,-1,0}^{(1)}\right\rangle\right),
\end{aligned}
$$

and

$$
\begin{aligned}
\left(\underline{\hat{\boldsymbol{H}}}_{3}^{(3)}\right)_{(2,-1,0),(3,-1,-1)}= & \left(\underline{\hat{\boldsymbol{H}}}_{3}^{(3)}\right)_{(3,-1,-1),(2,-1,0)}^{*} \\
= & \frac{b_{1}^{(12)} b_{2}^{(12)}\left(b_{1}^{(23)}\right)^{*}}{\left(E_{2,-1,0}^{(0)}-E_{1,-2,0}^{(0)}\right)\left(E_{3,-1,-1}^{(0)}-E_{2,-2,-1}^{(0)}\right)}+\frac{b_{1}^{(23)}\left(b_{1}^{(23)}\right)^{*} b_{2}^{(23)}}{\left(E_{2,-1,0}^{(0)}-E_{3,-2,0}^{(0)}\right)\left(E_{3,-1,-1}^{(0)}-E_{2,-2,1}^{(0)}\right)} \\
& +\frac{b_{2}^{(12)}\left(b_{1}^{(23)}\right)^{*} b_{1}^{(12)}}{\left(E_{2,-1,0}^{(0)}-E_{1,-1,-1}^{(0)}\right)\left(E_{3,-1,-1}^{(0)}-E_{2,-2,-1}^{(0)}\right)}+\frac{b_{2}^{(2)}\left(b_{2}^{(23)}\right)^{*} b_{2}^{(12)}}{\left(E_{2,-1,0}^{(0)}-E_{1,-1,-1}^{(0)}\right)\left(E_{3,-1,-1}^{(0)}-E_{2,-1,-2}^{(0)}\right)} \\
& +\frac{\left(b_{1}^{(23)}\right)^{*} b_{1}^{(23)} b_{2}^{(23)}}{\left(E_{2,-1,0}^{(0)}-E_{300}^{(0)}\right)\left(E_{3,-1,-1}^{(0)}-E_{2,0,-1}^{(0)}\right)}+\frac{b_{2}^{(12)} b_{1}^{(23)}\left(b_{1}^{(12)}\right)^{*}}{\left(E_{2,-1,0}^{(0)}-E_{1,-1,-1}^{(0)}\right)\left(E_{3,-1,-1}^{(0)}-E_{2,0,-1}^{(0)}\right)} \\
& -\frac{1}{2} \frac{\left(b_{1}^{(12)}\right)^{*} b_{2}^{(12)} b_{1}^{(23)}}{\left(E_{100}^{(0)}-E_{2,0,-1}^{(0)}\right)\left(E_{3,-1,-1}^{(0)}-E_{2,0,-1}^{(0)}\right)}-\frac{1}{2} b_{2}^{(23)}\left(\left\langle\phi_{2,-1,0}^{(1)} \mid \phi_{2,-1,0}^{(1)}\right\rangle+\left\langle\phi_{3,-1,-1}^{(1)} \mid \phi_{3,-1,-1}^{(1)}\right\rangle\right),
\end{aligned}
$$

where

$$
\begin{aligned}
\left\langle\phi_{100}^{(1)} \mid \phi_{100}^{(1)}\right\rangle= & \frac{\left|b_{2}^{(12)}\right|^{2}}{\left(E_{100}^{(0)}-E_{201}^{(0)}\right)^{2}}+\frac{\left|b_{1}^{(12)}\right|^{2}}{\left(E_{100}^{(0)}-E_{210}^{(0)}\right)^{2}}+\frac{\left|b_{2}^{(12)}\right|^{2}}{\left(E_{100}^{(0)}-E_{2,0,-1}^{(0)}\right)^{2}}, \\
\left\langle\phi_{2,-1,0}^{(1)} \mid \phi_{2,-1,0}^{(1)}\right\rangle= & \frac{\left|b_{2}^{(12)}\right|^{2}}{\left(E_{2,-1,0}^{(0)}-E_{1,-1,1}^{(0)}\right)^{2}}+\frac{\left|b_{1}^{(12)}\right|^{2}}{\left(E_{2,-1,0}^{(0)}-E_{1,-2,0}^{(0)}\right)^{2}}+\frac{\left|b_{2}^{(12)}\right|^{2}}{\left(E_{2,-1,0}^{(0)}-E_{1,-1,-1}^{(0)}\right)^{2}} \\
& \quad+\frac{\left|b_{2}^{(23)}\right|^{2}}{\left(E_{2,-1,0}^{(0)}-E_{3,-1,1}^{(0)}\right)^{2}}+\frac{\left|b_{1}^{(23)}\right|^{2}}{\left(E_{2,-1,0}^{(0)}-E_{300}^{0}\right)^{2}}+\frac{\left|b_{1}^{(23)}\right|^{2}}{\left(E_{2,-1,0}^{(0)}-E_{3,-2,0}^{(0)}\right)^{2}},
\end{aligned}
$$

and 


$$
\left\langle\phi_{3,-1,-1}^{(1)} \mid \phi_{3,-1,-1}^{(1)}\right\rangle=\frac{\left|b_{1}^{(23)}\right|^{2}}{\left(E_{3,-1,-1}^{(0)}-E_{2,0,-1}^{(0)}\right)^{2}}+\frac{\left|b_{1}^{(23)}\right|^{2}}{\left(E_{3,-1,-1}^{(0)}-E_{2,-2,-1}^{(0)}\right)^{2}}+\frac{\left|b_{2}^{(23)}\right|^{2}}{\left(E_{3,-1,-1}^{(0)}-E_{2,-1,-2}^{(0)}\right)^{2}} .
$$

The eigenfunctions and eigenvalues of the $3 \times 3$ effective Floquet Hamiltonian $\hat{H}_{3}$ are the approximate eigenfunctions, accurate to the first order, and approximate eigenvalues, accurate to the third order, corresponding to unperturbed states $|100\rangle,|2,-1,0\rangle$, and $|3,-1,-1\rangle$, of the Floquet Hamiltonian $\hat{H}_{F}$ of Eq. (7), i.e.,

$$
\begin{aligned}
& \left|\lambda_{100}\right\rangle=\left|\phi_{100}\right\rangle\left\langle\phi_{100} \mid \lambda_{100}\right\rangle+\left|\phi_{2,-1,0}\right\rangle\left\langle\phi_{2,-1,0} \mid \lambda_{100}\right\rangle+\left|\phi_{3,-1,-1}\right\rangle\left\langle\phi_{3,-1,-1} \mid \lambda_{100}\right\rangle, \\
& \left|\lambda_{2,-1,0}\right\rangle=\left|\phi_{100}\right\rangle\left\langle\phi_{100} \mid \lambda_{2,-1,0}\right\rangle+\left|\phi_{2,-1,0}\right\rangle\left\langle\phi_{2,-1,0} \mid \lambda_{2,-1,0}\right\rangle+\left|\phi_{3,-1,-1}\right\rangle\left\langle\phi_{3,-1,-1} \mid \lambda_{2,-1,0}\right\rangle,
\end{aligned}
$$

and

$$
\left|\lambda_{3,-1,-1}\right\rangle=\left|\phi_{100}\right\rangle\left\langle\phi_{100} \mid \lambda_{3,-1,-1}\right\rangle+\left|\phi_{2,-1,0}\right\rangle\left\langle\phi_{2,-1,0} \mid \lambda_{3,-1,-1}\right\rangle+\left|\phi_{3,-1,-1}\right\rangle\left\langle\phi_{3,-1,-1} \mid \lambda_{3,-1,-1}\right\rangle,
$$

where $\lambda$ 's and $|\lambda\rangle$ 's satisfy the eigenvalue equation

$$
\widehat{H}_{3}|\lambda\rangle=\lambda|\lambda\rangle \text {. }
$$

For a $3 \times 3$ Hermitian matrix $\underline{\hat{H}}_{3}=\underline{\hat{H}}_{3}^{\dagger}$, analytic expressions for its real eigenvalues $\lambda$ 's and components of corresponding eigenvectors $\left\langle\phi_{100} \mid \lambda\right\rangle,\left\langle\phi_{2,-1,0} \mid \lambda\right\rangle$, and $\left\langle\phi_{3,-1,-1} \mid \lambda\right\rangle$ can be obtained easily. ${ }^{24}$ We remark that once approximate results of $\left\{\lambda_{100},\left|\lambda_{100}\right\rangle\right\},\left\{\lambda_{2,-1,0},\left|\lambda_{2,-1,0}\right\rangle\right\}$, and $\left\{\lambda_{3,-1,-1},\left|\lambda_{3,-1,-1}\right\rangle\right\}$ are carried out, all other eigenvalues $\lambda_{\gamma k_{1} k_{2}}$ and eigenstates $\left|\lambda_{\gamma k_{1} k_{2}}\right\rangle$ of $\hat{H}_{F}$ can also be obtained, from the periodic structure of $\hat{H}_{F}$, to the same order of accuracy. Equation (5) can thus be approximated as

$$
\begin{aligned}
& F_{1 \alpha}(t)=\left[\left\langle\phi_{100} \mid \lambda_{\alpha m_{1} m_{2}}\right\rangle+C_{1 \alpha, 2}(t)\left\langle\phi_{2,-1,0} \mid \lambda_{\alpha m_{1} m_{2}}\right\rangle\right] \exp \left(-i \lambda_{\alpha m_{1} m_{2}} t\right), \\
& F_{2 \alpha}(t)=\left[\left\langle\phi_{2,-1,0} \mid \lambda_{\alpha m_{1} m_{2}}\right\rangle \exp \left(-i \omega_{1} t\right)+C_{2 \alpha, 1}(t)\left\langle\phi_{100} \mid \lambda_{\alpha m_{1} m_{2}}\right\rangle+C_{2 \alpha, 3}(t)\left\langle\phi_{3,-1,-1} \mid \lambda_{\alpha m_{1} m_{2}}\right\rangle\right] \exp \left(-i \lambda_{\alpha m_{1} m_{2}} t\right),
\end{aligned}
$$

and

$$
F_{3 \alpha}(t)=\left[\left\langle\phi_{3,-1,-1} \mid \lambda_{\alpha m_{1} m_{2}}\right\rangle \exp \left[-i\left(\omega_{1}+\omega_{2}\right) t\right]+C_{3 \alpha, 2}(t)\left\langle\phi_{2,-1,0} \mid \lambda_{\alpha m_{1} m_{2}}\right\rangle\right] \exp \left(-i \lambda_{\alpha m_{1} m_{2}} t\right),
$$

where

$$
\begin{aligned}
\left(\alpha, m_{1}, m_{2}\right) & \left\{\begin{array}{l}
(1,0,0) \text { if } \alpha=1 \\
(2,-1,0) \text { if } \alpha=2 \\
(3,-1,-1) \text { if } \alpha=3
\end{array}\right. \\
C_{1 \alpha, 2}(t)= & \frac{b_{2}^{(12)}}{E_{2,-1,0}^{(0)}-E_{1,-1,1}^{(0)}} e^{-i\left(\omega_{1}-\omega_{2}\right) t} \\
& +\frac{\left(b_{1}^{(12)}\right)^{*}}{E_{2,-1,0}^{(0)}-E_{1,-2,0}^{(0)}} e^{-2 i \omega_{1} t} \\
& +\frac{\left(b_{2}^{(12)}\right)^{*}}{E_{2,-1,0}^{(0)}-E_{1,-1,-1}^{(0)}} e^{-i\left(\omega_{1}+\omega_{2}\right) t}, \\
C_{2 \alpha, 1}(t)= & \frac{b_{2}^{(12)}}{E_{100}^{(0)}-E_{201}^{(0)} e^{i \omega_{2} t}+\frac{b_{1}^{(12)}}{E_{100}^{(0)}-E_{210}^{(0)}} e^{i \omega_{1} t}} \\
& +\frac{\left(b_{2}^{(12)}\right)^{*}}{E_{100}^{(0)}-E_{2,0,-1}^{(0)}} e^{-i \omega_{2} t}, \\
& +\frac{b_{1}^{(23)}}{C_{2 \alpha, 3}^{(t)}=} \\
& +\frac{\left(b_{1}^{(23)}\right)^{*}}{E_{3,-1,-1}^{(0)}-E_{2,-2,-1}^{(0)} b_{2}^{(23)}} e^{-i \omega_{2} t} \\
& E^{(0)-i\left(2 \omega_{1}+\omega_{2}\right) t}
\end{aligned}
$$

and

$$
\begin{aligned}
C_{3 \alpha, 2}(t)= & \frac{b_{2}^{(23)}}{E_{2,-1,0}^{(0)}-E_{3,-1,1}^{(0)}} e^{-i\left(\omega_{1}-\omega_{2}\right) t}+\frac{b_{1}^{(23)}}{E_{2,-1,0}^{(0)}-E_{300}^{(0)}} \\
& +\frac{\left(b_{1}^{(23)}\right)^{*}}{E_{2,-1,0}^{(0)}-E_{3,-2,0}^{(0)}} e^{-2 i \omega_{1} t} .
\end{aligned}
$$

By substituting Eqs. (29) into (4) one can write down the time evolution operator $\hat{U}\left(t ; t_{0}\right)$ in analytic form and thus can evaluate all relevant physical quantities, e.g., the probabilities of finding the system in a certain level, accordingly. The discussion of the time development can be greatly simplified in the rotating-wave frame ( not RWA) defined via the transformation

$$
\left|\Psi_{R}(t)\right\rangle=\hat{R}(t)|\Psi(t)\rangle,
$$

where the unitary transformation matrix $\underline{\hat{R}}(t)$ is defined by

$$
\underline{\hat{R}}(t) \equiv\left(\begin{array}{ccc}
1 & 0 & 0 \\
0 & e^{+i \omega_{1} t} & 0 \\
0 & 0 & e^{+i\left(\omega_{1}+\omega_{2}\right) t}
\end{array}\right) .
$$

In the rotating-wave picture, Eqs. (29) can be written as $F_{\beta \alpha}^{(R)}(t)=\left[\left\langle\phi_{\beta} \mid \lambda_{\alpha}\right\rangle+\sum_{\gamma} C_{\beta \alpha, \gamma}^{(R)}(t)\left\langle\phi_{\gamma} \mid \lambda_{\alpha}\right\rangle\right] e^{-i \lambda_{\alpha} t}$, 
where the abbreviated notations are defined as

$$
\begin{aligned}
& \left\langle\phi_{\beta} \mid \lambda_{\alpha}\right\rangle \equiv\left\langle\phi_{\beta n_{1} n_{2}} \mid \lambda_{\alpha m_{1} m_{2}}\right\rangle, \\
& \left\langle\phi_{\gamma} \mid \lambda_{\alpha}\right\rangle \equiv\left\langle\phi_{\gamma k_{1} k_{2}} \mid \lambda_{\alpha m_{1} m_{2}}\right\rangle,
\end{aligned}
$$

and

$$
\lambda_{\alpha} \equiv \lambda_{\alpha m_{1} m_{2}} .
$$

The nonvanishing coefficients $C_{\beta \alpha, \gamma}^{(R)}(t)$ 's are

$$
\begin{aligned}
& C_{1 \alpha, 2}^{(R)}(t)=C_{1 \alpha, 2}(t), \\
& C_{2 \alpha, 1(t)}^{(R)}=C_{2 \alpha, 1}(t) e^{i \omega_{1} t}, \\
& C_{2 \alpha, 3}^{(R)}(t)=C_{2 \alpha, 3}(t) e^{i \omega_{1} t},
\end{aligned}
$$

and

$$
C_{3 \alpha, 2}^{(R)}(t)=C_{3 \alpha, 2}(t) e^{i\left(\omega_{1}+\omega_{2}\right) t} .
$$

The time-evolution propagator $\widehat{U}^{(R)}(t ; 0)$ in the rotatingwave picture can thus be written as

$$
\begin{aligned}
\hat{U}_{\beta \alpha}^{(R)}(t ; 0)= & {[\underline{\hat{R}}(t) \underline{\hat{U}}(t ; 0)]_{\beta \alpha} } \\
= & \sum_{\gamma}\left[\left\langle\phi_{\beta} \mid \lambda_{\gamma}\right\rangle\left\langle\lambda_{\gamma} \mid \phi_{\alpha}\right\rangle+\sum_{\gamma^{\prime}}\left\{C_{\beta \gamma, \gamma^{\prime}}^{(R)}(t)\left\langle\phi_{\gamma^{\prime}} \mid \lambda_{\gamma}\right\rangle\left\langle\lambda_{\gamma} \mid \phi_{\alpha}\right\rangle+\left[C_{\alpha \gamma, \gamma^{\prime}}^{(R)}(0)^{*}\right]\left\langle\phi_{\beta} \mid \lambda_{\gamma}\right\rangle\left\langle\lambda_{\gamma} \mid \phi_{\gamma^{\prime}}\right\rangle\right\}\right. \\
& \left.\quad+\sum_{\gamma^{\prime}, \gamma^{\prime \prime}} C_{\beta \gamma, \gamma^{\prime}}^{(R)}(t)\left[C_{\alpha \gamma, \gamma^{\prime \prime}}^{(R)}(0)\right]^{*}\left\langle\phi_{\gamma^{\prime}} \mid \lambda_{\gamma}\right\rangle\left\langle\lambda_{\gamma} \mid \phi_{\gamma^{\prime \prime}}\right\rangle\right] e^{-i \lambda_{\gamma^{t}}} .
\end{aligned}
$$

Since the derivation above is formally a timeindependent perturbation theory, we can easily find out that the necessary condition for the GVV theory to be meaningful is that

$$
\left|\frac{\left\langle\chi_{p}^{(0)}\left|\hat{H}_{F}\right| \phi_{\alpha m_{1} m_{2}}^{(0)}\right\rangle}{E_{\alpha m_{1} m_{2}}^{(0)}-E_{p}^{(0)}}\right| \ll 1,
$$

where $\left|\chi_{p}^{(0)}\right\rangle$ 's are zeroth-order external-space wave functions and $\left|\phi_{\alpha m_{1} m_{2}}^{(0)}\right\rangle$ 's [with $\left(\alpha, m_{1}, m_{2}\right)$ can only be $(1,0,0),(2,-1,0)$, or $(3,-1,-1)]$ are the zeroth-order model-space wave functions defined in the GVV theory, see Eqs. (12) and (13).

For the type-I three-level system, Fig. 1(a), the condition (34) amounts to simple condition

$$
\left|b_{2}^{(23)} / \omega_{2}\right| \ll 1
$$

because of the large disparity between the two allowed transition frequencies $\left(E_{2}-E_{1}\right)$ and $\left(E_{3}-E_{2}\right)$. For the type-II three-level system, Fig. 1(b), the condition (34) implies that

$$
\left|\frac{b_{2}^{(12)}}{\left(\omega_{2}-\omega_{1}\right)-\Delta_{1}}\right| \ll 1
$$

and

$$
\left|\frac{b_{1}^{(23)}}{\left(\omega_{2}-\omega_{1}\right)+\Delta_{2}}\right| \ll 1,
$$

due to the small difference between $\left(E_{2}-E_{1}\right)$ and $\left(E_{3}-E_{2}\right)$.

From the above derivations we can easily speculate that the widely employed RWA approach is equivalent to a GVV degenerate perturbation theory of the lowest order under condition (34), i.e., the model-space wave functions are carried out only to the zeroth order, namely,

$$
\begin{aligned}
& \left|\phi_{100}\right\rangle=\left|\phi_{100}^{(0)}\right\rangle, \\
& \left|\phi_{2,-1,0}\right\rangle=\left|\phi_{2,-1,0}^{(0)}\right\rangle,
\end{aligned}
$$

and

$$
\left|\phi_{3,-1,-1}\right\rangle=\left|\phi_{3,-1,-1}^{(0)}\right\rangle
$$

and the $3 \times 3$ effective Floquet Hamiltonian $\hat{H}_{3}$ is evaluated only to the first order, namely,

$$
\hat{H}_{3}=\hat{H}_{3}^{(0)}+\hat{H}_{3}^{(1)} \text {, }
$$

where $\hat{H}_{3}^{(0)}$ and $\hat{H}_{3}^{(1)}$ are given in Eqs. (20) and (21). Unlike the traditional way of transforming the timedependent Schrödinger equation, i.e., Eq. (1), into a rotating-wave frame, i.e., Eq. (30), and neglecting all oscillatory parts in the transformed Hamiltonian, we thus have provided a different viewpoint on the applicability of the rotating-wave approximation. The RWA counterparts of Eqs. (32) and (33) can be obtained by simply replacing Eq. (19) by Eq. (39) and dropping out terms with coefficients $C_{\beta \alpha, \gamma}^{(R)}(t)$ of the first-order correction.

\section{NONLINEAR COHERENT VECTOR DESCRIPTION OF THREE-LEVEL SYSTEMS IN BICHROMATIC FIELDS}

Elgin $^{10}$ and Hioe and Eberly ${ }^{9,12}$ have shown that a classical vector description of any three-level system in the presence of laser fields can be obtained based on the eight generators of the SU(3) group. Hioe and Eberly ${ }^{12}$ further demonstrated that, within the RWA, the eightdimensional coherence vector can be factored into several independent vectors of smaller dimensions, thus revealing additional dynamical symmetries, under the following two conditions: (i) two-photon resonance and the same timedependence of field amplitudes, or (ii) equal detunings and equal couplings. The purpose of this section is to define specifically the set of eight generators of the SU(3) group on which our calculations, which are not restricted to the $R W A$, will be finally based.

We shall adopt the standard form of the SU(3) generators used by Gell-Mann, ${ }^{26}$ namely, 


$$
\widehat{\mathfrak{s}} \equiv\left\{\hat{\boldsymbol{y}}_{i} \mid i=1,2, \ldots, 8\right),
$$

where

$$
\begin{aligned}
& \underline{\hat{\underline{s}}}_{1}=\left(\begin{array}{lll}
0 & 1 & 0 \\
1 & 0 & 0 \\
0 & 0 & 0
\end{array}\right), \quad \underline{\hat{\underline{1}}}_{2}=\left(\begin{array}{ccc}
0 & -i & 0 \\
i & 0 & 0 \\
0 & 0 & 0
\end{array}\right), \quad \underline{\hat{\underline{\hat{s}}}}_{3}=\left(\begin{array}{ccc}
1 & 0 & 0 \\
0 & -1 & 0 \\
0 & 0 & 0
\end{array}\right) \text {, } \\
& \underline{\hat{\underline{s}}}_{4}=\left(\begin{array}{lll}
0 & 0 & 1 \\
0 & 0 & 0 \\
1 & 0 & 0
\end{array}\right), \quad \underline{\hat{s}}_{5}=\left(\begin{array}{ccc}
0 & 0 & -i \\
0 & 0 & 0 \\
i & 0 & 0
\end{array}\right), \quad \hat{\underline{s}}_{6}=\left(\begin{array}{lll}
0 & 0 & 0 \\
0 & 0 & 1 \\
0 & 1 & 0
\end{array}\right) \text {, } \\
& \underline{\hat{\underline{s}}}_{7}=\left(\begin{array}{ccc}
0 & 0 & 0 \\
0 & 0 & -i \\
0 & i & 0
\end{array}\right), \quad \underline{\hat{\underline{s}}}_{8}=\frac{1}{\sqrt{3}}\left(\begin{array}{ccc}
1 & 0 & 0 \\
0 & 1 & 0 \\
0 & 0 & -2
\end{array}\right) \text {. }
\end{aligned}
$$

In terms of the eight $3 \times 3$ matrices in Eq. (40) and the $3 \times 3$ identity matrix $\underline{\underline{I}}$, the Hamiltonian in Eq. (2) can be expressed as

$$
\underline{\hat{H}}(t)=\frac{1}{3}\left(\sum_{\alpha=1}^{3} E_{\alpha}\right) \hat{\underline{I}}+\frac{1}{2} \sum_{j=1}^{8} \Gamma_{j}(t) \underline{\hat{s}}_{j},
$$

and the density matrix of the system can be written as

$$
\hat{\underline{\rho}}(t)=\frac{1}{3} \hat{I}+\frac{1}{2} \sum_{j=1}^{8} S_{j}(t) \underline{\hat{\hat{s}}}_{j},
$$

where

$$
\Gamma_{j}(t)=\operatorname{Tr}\left(\underline{\hat{H}}_{\hat{\hat{s}}_{j}}\right),
$$

and

$$
S_{j}(t)=\operatorname{Tr}\left[\underline{\rho}^{(t)} \underline{\hat{s}}_{j}\right] .
$$

Substituting Eqs. (41) and (42) into the Liouville equation

$$
i \frac{\partial \hat{\rho}(t)}{\partial t}=[\hat{H}(t), \hat{\rho}(t)]
$$

results in an equation of motion for the coherence vector $\mathbf{S}(t)$, namely,

$$
\frac{d}{d t} \mathbf{S}_{j}=\sum_{k, l} f_{j k l} \Gamma_{k}(t) \mathbf{S}_{l},
$$

where $f_{j k l}$ 's are the structure constants associated with the Gell-Mann-type generators, i.e., Eqs. (40) of the group $\mathrm{SU}(3)$. The length of the coherent vector $\mathbf{S}(t)$ is a constant of motion and can be evaluated readily, i.e., $K \equiv|\mathbf{S}|^{2}=2\left[\operatorname{Tr}(\underline{\hat{\rho}} \underline{\hat{\rho}})-\frac{1}{3}\right]$.

At exact two-photon resonance condition, i.e., $\Delta_{1}+\Delta_{2}=0$, within the RWA, while the equation of motion, Eq. (46), for the coherence vector defined in Eqs. (40) and (44) cannot be further factored into smaller independent subvectors, Hioe and Eberly ${ }^{16}$ have shown that by regrouping the components of $\mathbf{S}(t)$ into three subvectors, i.e.,

$$
\mathbf{S}(t)=\mathbf{A}(t)+\mathbf{B}(t)+\mathbf{C}(t),
$$

where

$$
A_{1}(t)=\frac{1}{b}\left[\Omega_{1} S_{1}(t)+\Omega_{2}^{\prime} S_{6}(t)\right],
$$

$$
\begin{aligned}
& A_{2}(t)=-\frac{1}{b}\left[\Omega_{1} S_{2}(t)-\Omega_{2}^{\prime} S_{7}(t)\right] \\
& \begin{aligned}
A_{3}(t)=\frac{1}{2} b^{-2}\left\{\left[2 \Omega_{1}^{2}+\left(\Omega_{2}^{\prime}\right)^{2}\right] S_{3}(t)-\sqrt{3}\left(\Omega_{2}^{\prime}\right)^{2} S_{8}(t)\right. \\
\left.\quad+2 \Omega_{1}^{2}\left(\Omega_{2}^{\prime}\right)^{2} S_{4}(t)\right\}
\end{aligned} \\
& \begin{aligned}
B_{1}(t) & =S_{5}(t)
\end{aligned} \\
& B_{2}(t)=b^{-1}\left[\Omega_{2}^{\prime} S_{1}(t)-\Omega_{1} S_{6}(t)\right] \\
& B_{3}(t)=b^{-1}\left[\Omega_{2}^{\prime} S_{2}(t)+\Omega_{1} S_{7}(t)\right] \\
& B_{4}(t)=b^{-2}\left\{\Omega_{1} \Omega_{2}^{\prime} S_{3}(t)+\sqrt{3} \Omega_{1} \Omega_{2}^{\prime} S_{8}(t)\right. \\
& \left.\quad-\left[\Omega_{1}^{2}-\left(\Omega_{2}^{\prime}\right)^{2}\right] S_{4}(t)\right\}
\end{aligned}
$$

and

$$
\begin{gathered}
C(t)=\frac{1}{2} b^{-2}\left\{\sqrt{3}\left(\Omega_{2}^{\prime}\right)^{2} S_{3}(t)-\left[2 \Omega_{1}^{2}-\left(\Omega_{2}^{\prime}\right)^{2}\right] S_{8}(t)\right. \\
\left.-2 \sqrt{3} \Omega_{1} \Omega_{2}^{\prime} S_{4}(t)\right\}
\end{gathered}
$$

with

$$
b=\left[\Omega_{1}^{2}+\left(\Omega_{2}^{\prime}\right)^{2}\right]^{1 / 2},
$$

the subvectors $\mathbf{A}(t), \mathbf{B}(t)$, and $\mathbf{C}(t)$, of dimensions three, four, and one, rotate independently, and their respective length preserves in the course of the time. Under these conditions, the analytic expressions for the case of the constant field amplitudes and field frequencies can be derived easily from the decoupled equations of motion for subvectors $\mathbf{A}(t), \mathbf{B}(t)$, and $\mathbf{C}(t)$, respectively. Assuming that (i) the system is in its ground state initially, and (ii) the initial phases $\theta_{1}$ and $\theta_{2}$ of the fields are zero, components of $\mathbf{A}(t), \mathbf{B}(t)$, and $\mathbf{C}(t)$ can be written as

$$
\begin{aligned}
& A_{1}(t)=-\frac{2 \Omega_{1}^{2} \Delta}{b p^{2}}[1-\cos (p t)], \\
& A_{2}(t)=\frac{2 \Omega_{1}^{2}}{b p} \sin (p t), \\
& A_{3}(t)=-\frac{4 \Omega_{1}^{2}}{p^{2}}[1-\cos (p t)]+\frac{\Omega_{1}^{2}}{b^{2}}, \\
& B_{1}(t)=-\frac{2 \Omega_{1} \Omega_{2}^{\prime}}{p}\left[\frac{1}{\xi} \sin (\xi t)-\frac{1}{\eta} \sin (\eta t)\right],
\end{aligned}
$$

$B_{2}(t)=\frac{2 \Omega_{1} \Omega_{2}^{\prime}}{b p}[\cos (\xi t)-\cos (\eta t)]$,

$$
\begin{array}{r}
B_{3}(T)=\frac{2 \Omega_{1} \Omega_{2}^{\prime}}{b p \Delta}\left[-\xi\left(1-\frac{b^{2}}{\xi^{2}}\right) \sin (\xi t)\right. \\
\left.+\eta\left(1-\frac{b^{2}}{\eta^{2}}\right) \sin (\eta t)\right],
\end{array}
$$

$$
\begin{aligned}
B_{4}(t)=\frac{2 \Omega_{1} \Omega_{2}^{\prime}}{b^{2}}+\frac{2 \Omega_{1} \Omega_{2}^{\prime}}{p \Delta}[ & -\left(1-\frac{b^{2}}{\xi^{2}}\right)[1-\cos (\xi t)] \\
& \left.+\left(1-\frac{b^{2}}{\eta^{2}}\right)[1-\cos (\eta t)]\right],
\end{aligned}
$$

and 


$$
C(t)=\frac{2\left(\Omega_{2}^{\prime}\right)^{2}-\Omega_{1}^{2}}{\sqrt{3} b^{2}} \equiv \sqrt{K_{3}},
$$

where

$$
\begin{aligned}
& \Delta=\Delta_{1}=-\Delta_{2} \neq 0, \\
& p=\left(\Delta^{2+4} b^{2}\right)^{1 / 2},
\end{aligned}
$$

and

$$
\xi=\frac{1}{2}(p+\Delta),
$$

and

$$
\eta=\frac{1}{2}(p-\Delta) .
$$

Here we note that (i) initial conditions of $\mathbf{A}(t), \mathbf{B}(t)$, and $\mathrm{C}(t)$ have been integrated into Eqs. (50) via (52), and (ii) same expressions $A_{1}(t), A_{2}(t), A_{3}(t)$, and $B_{1}(t)$ were also obtained by Brewer and Hahn in their previous studies of Raman beats and two-photon absorption and emission. ${ }^{4}$ From Eqs. (50) and (51), and the conservation of the lengths of vectors $\mathbf{A}(t)$ and $\mathbf{B}(t)$, we can see that the vector $\mathbf{A}(t)$ pursues a circular motion defined by the relations

$$
\frac{b^{2} p^{2}}{4 \Omega_{1}^{4}}\left[\frac{p^{2}}{\Delta^{2}}\left[A_{1}(t)+\frac{2 \Omega_{1}^{2} \Delta}{b p^{2}}\right]^{2}+\left[A_{2}(t)\right]^{2}\right]=1,
$$

and

$$
\left[A_{1}(t)\right]^{2}+\left[A_{2}(t)\right]^{2}+\left[A_{3}(t)\right]^{2}=\frac{\Omega_{1}^{4}}{b^{4}} \equiv K_{1} .
$$

Yet the vector $\mathbf{B}(t)$ engages in a much more complicated motion defined by the relations

$$
\begin{aligned}
& B_{2}(t)^{2}+B_{3}(t)^{2}=\frac{8 \Omega_{1}^{2}\left(\Omega_{2}^{\prime}\right)^{2}}{b^{2} p^{2}}[1-\cos (p t)], \\
& B_{1}(t)^{2}+B_{4}(t)^{2}=\frac{4 \Omega_{1}^{2}\left(\Omega_{2}^{\prime}\right)^{2}}{b^{4}}\left(1-\frac{2 b^{2}}{p^{2}}[1-\cos (p t)]\right),
\end{aligned}
$$

and

$$
\left[B_{1}(t)\right]^{2}+\left[B_{2}(t)\right]^{2}+\left[B_{3}(t)\right]^{2}+\left[B_{4}(t)\right]^{2}=\frac{4 \Omega_{1}^{2}\left(\Omega_{2}^{\prime}\right)^{2}}{b^{4}} \equiv K_{2},
$$

It is thus clear that the projection of the trajectory of $\mathbf{A}(t)$ on either the $A_{1}-A_{2}$, Eq. (53a), or the $A_{1}-A_{3}$ plane is an ellipse, whereas projections of the trajectory of $\mathbf{B}(t)$ on $B_{2}-B_{3}$ and $B_{1}-B_{4}$ planes, respectively, are spinning cardioid and cycloid characterized by the frequency $p$. Note that at the exact two-photon resonance $\Delta_{1}=-\Delta_{2}=\Delta$, but $\Delta \neq 0$, in the RWA limit, the three quasienergies are $0, \xi$, and $-\eta$ associated, respectively, with the quasienergy states $\left|\lambda_{100}\right\rangle$ (or $\left|\lambda_{3,-1,-1}\right\rangle$ depending on Rabi frequencies $\Omega_{1}$ and $\Omega_{2}^{\prime}$, and also on the sign of the detuning $\Delta$ if beyond the RWA limit), $\left|\lambda_{2,-1,0}\right\rangle$, and $\left|\lambda_{3,-1,1}\right\rangle$ (or $\left.\left|\lambda_{100}\right\rangle\right)$. The quantity $p$ is simply the difference between quasienergies $\lambda_{2,-1,0}$ and $\lambda_{100}$ (or $\lambda_{3,-1,-1}$ ), and is predominantly of one-photon character. Therefore, we can see that the subcoherence vector $\mathbf{A}(t)$ depicts the coherence between the quasienergy states $\left|\lambda_{2,-1,0}\right\rangle$ and $\left|\lambda_{100}\right\rangle$ (or $\left|\lambda_{3,-1,-1}\right\rangle$ ), while the vector $\mathbf{B}(t)$ describes the coherences between the rest of two pairs of the quasienergy states $\left|\lambda_{2,-1,0}\right\rangle$ and $\left|\lambda_{3,-1,-1}\right\rangle$ (or $\left|\lambda_{100}\right\rangle$ ), and $\left|\lambda_{100}\right\rangle$ and $\left|\lambda_{3,-1,-1}\right\rangle$. As pointed out by Hioe and Eberly, the subvector $\mathbf{B}(t)$ can be further decoupled into two two-dimensional vectors $b_{1}(t)=\left(B_{1}, B_{2}\right)$ and $b_{2}(t)=\left(B_{3}, B_{4}\right)$ if the one-photon detunings $\Delta_{1}=\Delta_{2}=0$. With the same initial conditions as those in Eqs. (50) via (52), one can easily find that, at $\Delta_{1}=\Delta_{2}=0$,

$$
\begin{aligned}
& B_{1}(t)=B_{2}(t)=0, \quad B_{3}(t)=2 \Omega_{1} \Omega_{2}^{\prime} b^{-2} \sin (b t), \\
& B_{4}(t)=2 \Omega_{1} \Omega_{2}^{\prime} b^{-2} \cos (b t) .
\end{aligned}
$$

In more general cases deviations from either the RWA limit, or the two-photon resonance condition will modify the trajectory of the $S(t)$ described by Eqs. (50) via (53) and thus break the dynamic symmetries embodied in the independence of the subvectors $\mathbf{A}, \mathbf{B}$, and $\mathbf{C}$ in the course of the time. The study of this symmetry-breaking effect can be facilitated by means of the perturbation scheme, which includes all the first-order corrections beyond the RWA (Sec. III). Alternatively, one can approach this by looking into the exact equation of motion for the coherent vector $\mathbf{S}$ in terms of the subvectors $\mathbf{A}, \mathbf{B}$, and $\mathbf{C}$ :

$$
\frac{d \mathbf{S}}{d t}=\underline{\Lambda} \mathbf{S}
$$

where $\mathbf{S}$ is the column vector of dimension eight,

$$
\mathrm{S}=\left(A_{1}, A_{2}, A_{3}, B_{1}, B_{2}, B_{3}, B_{4}, C\right)^{T},
$$

and $\Lambda$ is the antisymmetric $8 \times 8$ matrix defined by

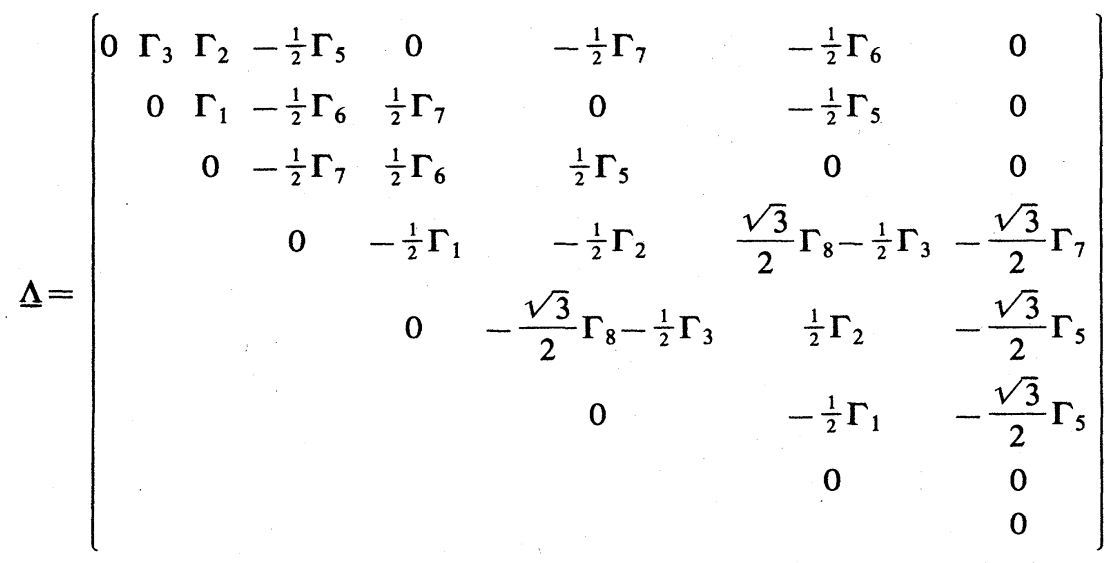


with coefficients

$$
\begin{aligned}
& \Gamma_{1}(t)=(2 / b)\left(b^{2}+\Omega_{1}^{2} \cos \left(2 \omega_{1} t\right)+\left(\Omega_{2}^{\prime}\right)^{2} \cos \left(2 \omega_{2} t\right)+\left(\Omega_{1} \Omega_{2}+\Omega_{1}^{\prime} \Omega_{2}^{\prime}\right)\left\{\cos \left[\left(\omega_{1}-\omega_{2}\right) t\right]+\cos \left[\left(\omega_{1}+\omega_{2}\right) t\right]\right\}\right), \\
& \Gamma_{2}(t)=(2 / b)\left\{\Omega_{1}^{2} \sin \left(2 \omega_{1} t\right)-\left(\Omega_{2}^{\prime}\right)^{2} \sin \left(2 \omega_{2} t\right)+\left(\Omega_{1} \Omega_{2}+\Omega_{1}^{\prime} \Omega_{2}^{\prime}\right) \sin \left[\left(\omega_{1}-\omega_{2}\right) t\right]+\left(\Omega_{1} \Omega_{2}-\Omega_{1}^{\prime} \Omega_{2}^{\prime}\right) \sin \left[\left(\omega_{1}+\omega_{2}\right) t\right]\right\}, \\
& \Gamma_{3}(t)=b^{-2}\left[-b^{2} \Delta_{1}+\left(\Omega_{2}^{\prime}\right)^{2}\left(\Delta_{1}+\Delta_{2}\right)\right], \\
& \Gamma_{4}(t)=0, \\
& \Gamma_{5}(t)=\left(2 / b^{2}\right)\left(\Omega_{1} \Omega_{2}^{\prime}\left[\cos \left(2 \omega_{1} t\right)-2 \cos \left(2 \omega_{2} t\right)\right]+\left(\Omega_{2} \Omega_{2}^{\prime}-\Omega_{1} \Omega_{1}^{\prime}\right)\left\{\cos \left[\left(\omega_{1}-\omega_{2}\right) t\right]+\cos \left[\left(\omega_{1}+\omega_{2}\right) t\right]\right\}\right), \\
& \Gamma_{6}(t)=-\left(2 / b^{2}\right)\left\{\Omega_{1} \Omega_{2}^{\prime}\left[\sin \left(2 \omega_{1} t\right)+\sin \left(2 \omega_{2} t\right)\right]+\left(\Omega_{2} \Omega_{2}^{\prime}-\Omega_{1} \Omega_{1}^{\prime}\right) \sin \left[\left(\omega_{1}-\omega_{2}\right) t\right]-\left(\Omega_{2} \Omega_{2}^{\prime}+\Omega_{1} \Omega_{1}^{\prime}\right) \sin \left[\left(\omega_{1}+\omega_{2}\right) t\right]\right\}, \\
& \Gamma_{7}(t)=-\left(2 / b^{2}\right) \Omega_{1} \Omega_{2}^{\prime}\left(\Delta_{1}+\Delta_{2}\right),
\end{aligned}
$$

and

$$
\Gamma_{8}(t)=\frac{1}{3} b^{-2}\left\{-b^{2} \Delta_{1}+\left[2 \Omega_{1}^{2}-\left(\Omega_{2}^{\prime}\right)^{2}\right]\left(\Delta_{1}+\Delta_{2}\right)\right\} .
$$

Here we have presented Eq. (56) in the rotating-wave frame. It is easy to see that Eqs. (56) reduce to the three decoupled equations of the motion derived by Hioe and Eberly in the case of the exact two-photon resonance, i.e., $\Delta_{1}+\Delta_{2}=0$, within the RWA. As the non-RWA terms, which are signified by time-dependent factors and the total detuning $\Delta_{1}+\Delta_{2}$ in Eqs. (57), become more significant, not only the vectors $\mathbf{A}, \mathbf{B}$, and $\mathbf{C}$, defined in (47)-(49), become less independent of each other, but also their evolutions in the course of the time become more complicated. The dynamical symmetries embodied in the twophoton resonant three-level system within the RWA can, therefore, only be at best approximate in nature, and the underlying richness of the evolution of the system can

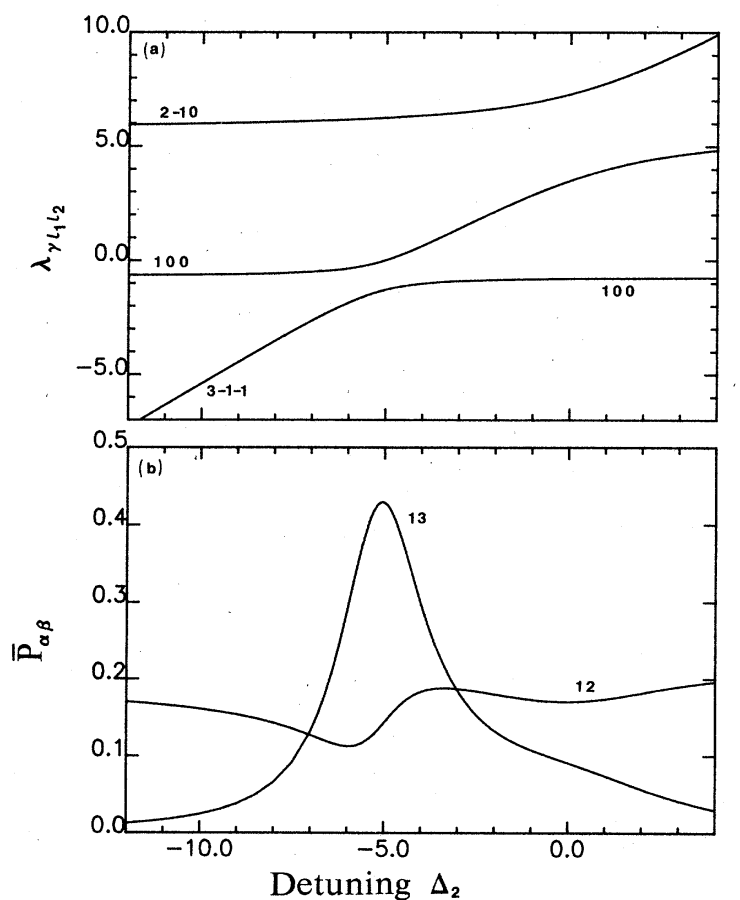

FIG. 2. (a) Quasienergies and (b) long time-averaged transition probabilities $\bar{P}_{12}$ and $\bar{P}_{13}$ as functions of the detuning $\Delta_{2}$ at $E_{2}-E_{1}=4000.0$ (arbitrary units), $E_{3}-E_{2}=50.0, \Delta_{1}=5.0$, and $\Omega_{1}=\Omega_{1}^{\prime}=\Omega_{2}=\Omega_{2}^{\prime}=2.0$ of the type-I three-level system. only be understood by going beyond the RWA limit. While we can achieve this goal by directly solving the time-dependent coupled equation (54) numerically, it is far more expedient to employ the time-independent GVV perturbation theory, i.e., Sec. III, to exploit the intricate symmetry-breaking effects in the dynamical evolution of
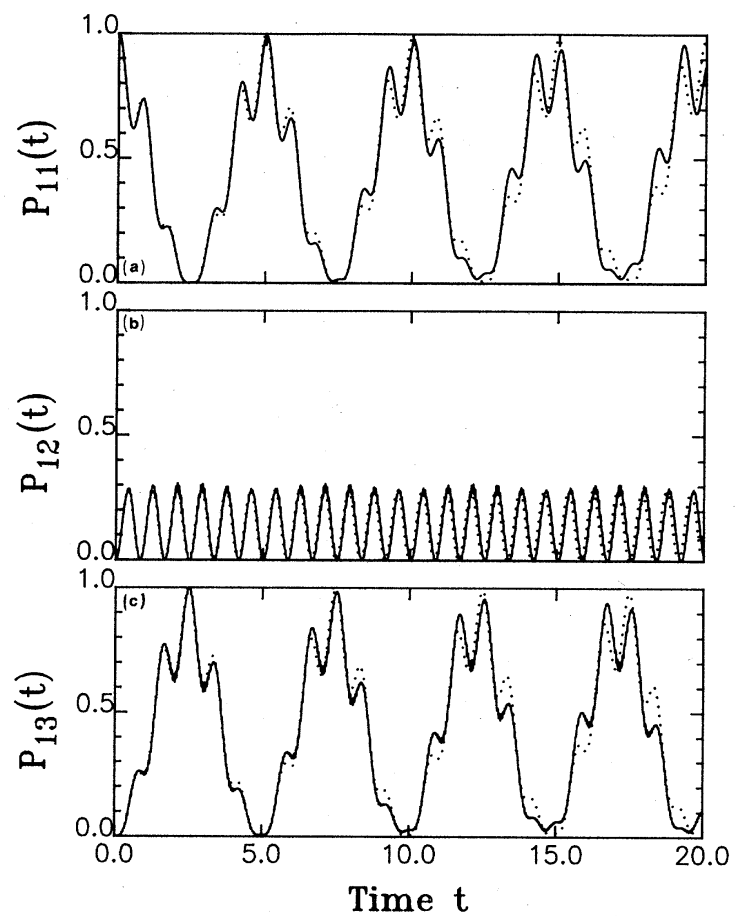

FIG. 3. Time-dependent transition probabilities (a) $P_{11}(t)$, (b) $P_{12}(t)$, and (c) $P_{13}(t)$ for the type-I cascade three-level system at $\left(E_{2}-E_{1}\right)=4000.0, \quad\left(E_{3}-E_{2}\right)=50.0, \quad \Delta_{1}=-\Delta_{2}=5.0, \quad$ and $\Omega_{1}=\Omega_{1}^{\prime}=\Omega_{2}=\Omega_{2}^{\prime}=2.0$. Two time periods characterizing the population oscillations are defined in terms of reciprocals of the differences between the quasienergies $\lambda_{100}$ and $\lambda_{2,-1,0}$, and $\lambda_{100}$ and $\lambda_{3,-1,-1}$, i.e., the one-photon flopping period $T_{12}=2 \pi /$ $\left|\lambda_{100}-\lambda_{2,-1,0}\right|$ and the two-photon flopping period $T_{13}=2 \pi /$ $\left|\lambda_{100}-\lambda_{3,-1,-1}\right|$. In the RWA calculations, dotted curves, $T_{12}^{(\mathrm{RWA})}=0.8322$ and $T_{13}^{(\mathrm{RWA})}=4.9283$, while in the $\operatorname{GVV}(3,1)$ calculations, solid curves, $T_{12}=0.8362$ and $T_{13}=4.8866$. Time development of the first four two-photon flopping periods is shown in this case. 
TABLE I. Comparison of the quasienergy calculations for the cascade three-level system [type I, Fig. 1(a)] at exact two-photon resonance condition. Parameters used are $E_{1}=0, E_{2}-E_{1}=4000.0$, $E_{3}-E_{2}=50.0, \Delta_{1}=-\Delta_{2}=5.0$, and $\Omega_{1}=\Omega_{1}^{\prime}=\Omega_{2}=\Omega_{2}^{\prime}=2.0$ (arbitrary units).

\begin{tabular}{lcccc}
\hline \hline & Exact [2,2] & Exact [1,1] & GVV(3,1) & RWA \\
\hline$\lambda_{100}$ & -1.2668 & -1.2667 & -1.2667 & -1.2749 \\
$\lambda_{2,-1,0}$ & 6.2477 & 6.2477 & 6.2477 & 6.2749 \\
$\lambda_{3,-1,-1}$ & 0.0190 & 0.0191 & 0.0191 & 0.0 \\
\hline \hline
\end{tabular}

the coherent vector.

The expectation value of an arbitrary physical operator $\widehat{O}$ can be evaluated via the definition

$$
\langle\hat{O}\rangle \equiv\langle\Psi(t)|\hat{O}| \Psi(t)\rangle=\operatorname{Tr}[\hat{\rho}(t) \hat{O}] .
$$

Here the density-matrix operator $\hat{\rho}(t)$, in the rotatingwave frame,

$$
\hat{\rho}(t)=\widehat{U}^{(R)}(t ; 0) \hat{\rho}(0)\left[\hat{U}^{(R)}(t ; 0)\right]^{\dagger}
$$

is expressed in terms of the evolution propagator $\widehat{U}^{(R)}(t ; 0)$, defined in Eq. (33), and the initial-state density matrix $\hat{\rho}(0)$ of the three-level system. The analytic expressions for components of $\mathbf{A}, \mathbf{B}$, and $\mathbf{C}$ can thus be derived by employing Eqs. (59), (44), and (47)-(49).

\section{RESULTS AND DISCUSSIONS}

In this section we shall present the results for the following three different cases: (1) $\left(E_{2}-E_{1}\right) \gg\left(E_{3}-E_{2}\right)$, $\Omega_{1}=\Omega_{2}=\Omega_{1}^{\prime}=\Omega_{2}^{\prime}$, and $\Delta_{1}=-\Delta_{2}=\Delta$, corresponding to a

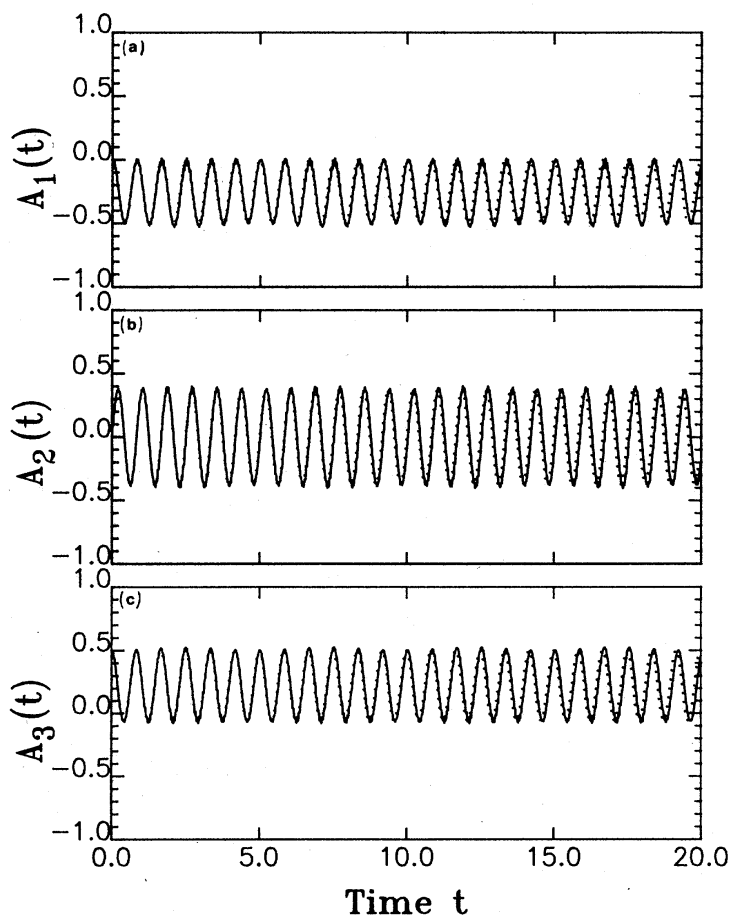

FIG. 4. Components of the subvector $\mathrm{A}(t)$, i.e., (a) $A_{1}(t)$, (b) $A_{2}(t)$, and (c) $A_{3}(t)$, as functions of time $t$. Parameters same as shown in Fig. 3. two-photon resonance case of the type-I three-level system, Fig. 1(a); (2) $\left|\left(E_{2}-E_{1}\right)-\left(E_{3}-E_{2}\right)\right|<\left(E_{2}-E_{1}\right)$ and $\left(E_{3}-E_{2}\right), \Omega_{1}=\Omega_{1}^{\prime} \neq \Omega_{2}=\Omega_{2}^{\prime}$, and $\Delta_{1}=-\Delta_{2}=\Delta$, a two-photon resonance case of the type-II three-level system, Fig. 1(b); and finally (3) $\left|\left(E_{2}-E_{1}\right)-\left(E_{3}-E_{2}\right)\right|$ $\ll\left(E_{2}-E_{1}\right)$ and $\left(E_{3}-E_{2}\right), \quad \Omega_{1}=\Omega_{2}=\Omega_{1}^{\prime}=\Omega_{2}^{\prime}$, and $\Delta_{1} \neq-\Delta_{2}$, a nonresonance case of the type-II three-level system, Fig. 1(b). In all these cases we shall assume that (i) the initial phases $\theta_{1}$ and $\theta_{2}$ of the laser fields are zero; (ii) the system is initially in its ground level, i.e., level 1; and (iii) $\left|\Delta_{1}\right| \ll\left(E_{2}-E_{1}\right)$ and $\left|\Delta_{2}\right| \ll\left(E_{3}-E_{2}\right)$. Thus the RWA two-photon resonance processes are the dominant channel responsible for the dynamics of the system in the presence of two laser fields. In the results to be presented in this section we shall also show the influence of various symmetry-breaking mechanisms, namely, the two-photon off resonance and the non- $R W A$ terms, on the dynamic symmetries inherited from the assumption of the exact two-photon resonance in the RWA limit. Calculations made by solving numerically the (converged) Floquet eigenvalue equation, i.e., Eq. (6) will be denoted as exact

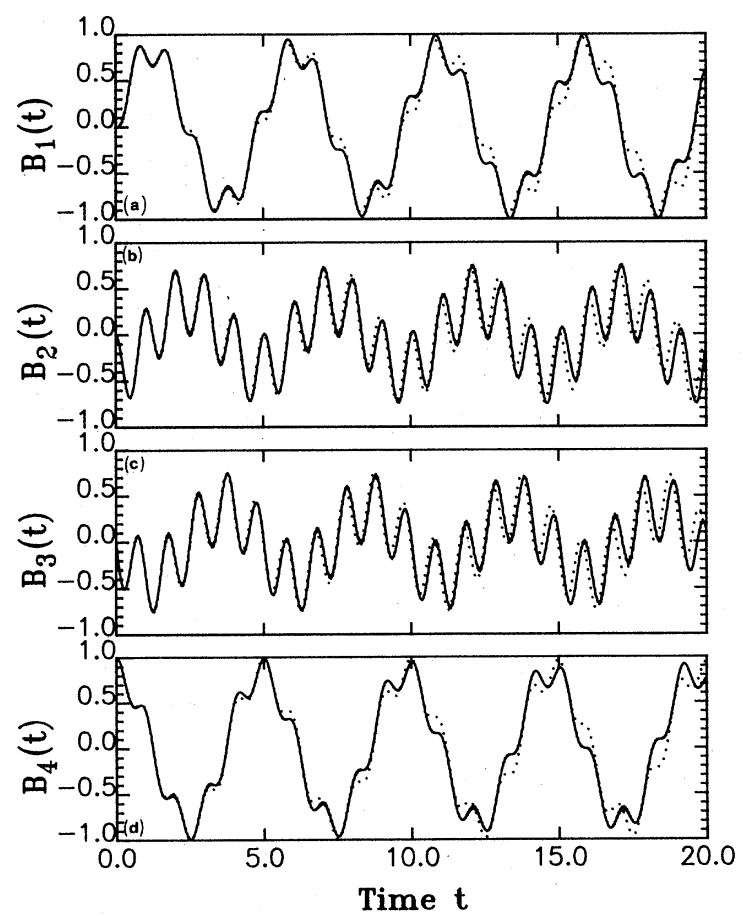

FIG. 5. Components of the subvector $\mathbf{B}(t)$, i.e., (a) $B_{1}(t)$, (b) $B_{2}(t)$, (c) $B_{3}(t)$, as functions of time $t$. Parameters same as shown in Fig. 3. 

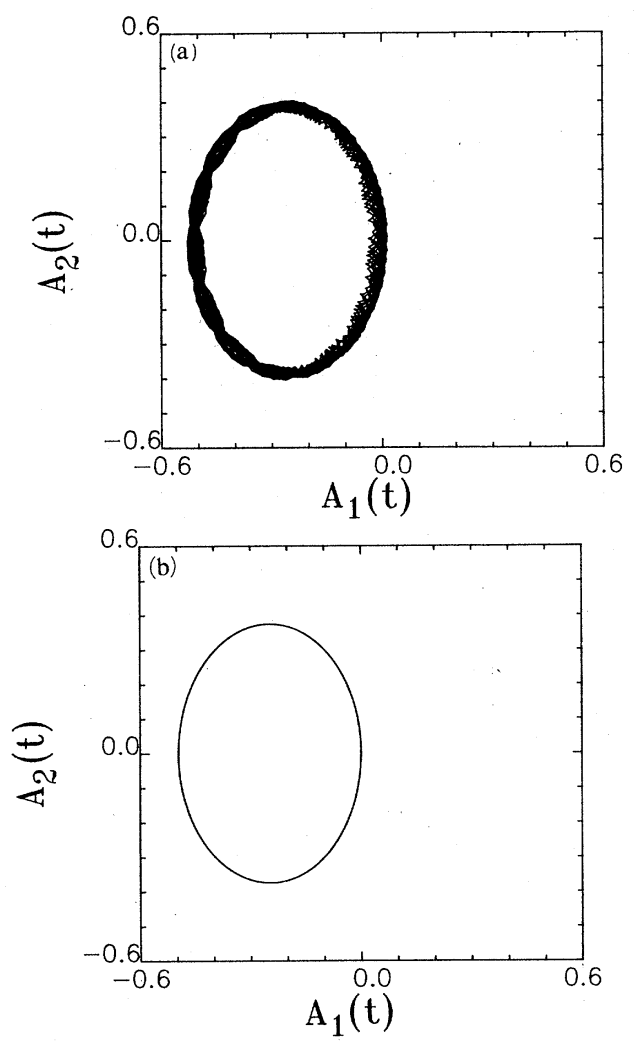

FIG. 6. Projection of the trajectory of the subvector $\mathbf{A}(t)$ on the $A_{1}-A_{2}$ plane for the same duration as shown in Fig. 4. (a) $\operatorname{GVV}(3,1)$ results and (b) RWA results.
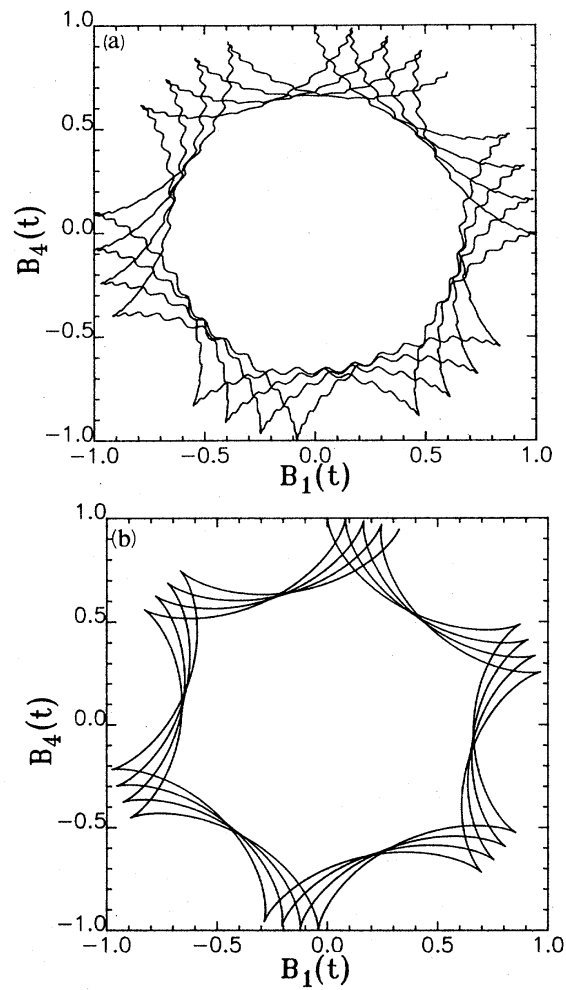

FIG. 7. Projection of the trajectory of the subvector $\mathbf{B}(t)$ on the $B_{1}-B_{4}$ plane for the same case and time duration as shown in Fig. 5. (a) GVV $(3,1)$ results and (b) RWA results.
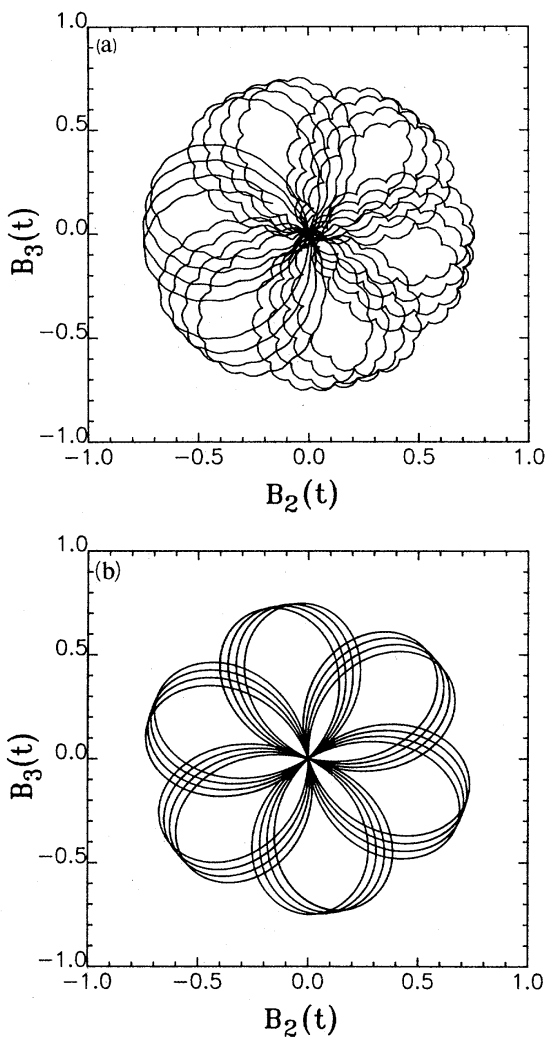

FIG. 8. Projection of the trajectory of the subvector $\mathbf{B}(t)$ on the $B_{2}-B_{3}$ plane for the same case and time duration as shown in Fig. 5. (a) $\operatorname{GVV}(3,1)$ results and (b) RWA results.

[ $N_{1}, N_{2}$ ] where $N_{1}$ and $N_{2}$ indicate the Floquet Hamiltonian, Eq. (7), is a truncated matrix comprising photon blocks $N_{1}, N_{1}-1, \ldots,-N_{1}$ from the first laser field and photon blocks $N_{2}, N_{2}-1, \ldots,-N_{2}$ from the second field. Calculations using the GVV perturbation theory will be denoted by $\operatorname{GVV}(3,1)$ with the notation $(3,1)$ meaning that the eigenvalues are carried out to the third order, i.e., Eq. (19), and the model-space wave function to the first order, i.e., Eqs. (18). The RWA results, which are equivalent to a GVV $(1,0)$ calculation are simply denoted as RWA. Arbitrary units shall be used and all results in the following are to be presented in the rotating-wave frame.

\section{A. Type-I three-level system at exact two-photon resonance}

Here we specifically choose that $E_{2}-E_{1}=4000.0$, $E_{3}-E_{2}=50.0, \Delta_{1}=\Delta_{2}=-5.0$, and $\Omega_{i}=\Omega_{i}^{\prime}=2.0$ with $i=1,2$. In Table I we tabulate the quasienergies, $\lambda_{100}$, $\lambda_{2,-1,0}$, and $\lambda_{3,-1,-1}$, of various versions of calculations. It is found that for this case the exact results converge at photon blocks $[2,2]$. The $\operatorname{GVV}(3,1)$ results agree very well with the exact ones, while the RWA ones deviate slightly. The quasienergies and the long time-averaged transition probabilities $\bar{P}_{12}$ and $\bar{P}_{13}$, Eq. (11b), as functions of the detuning $\Delta_{2}$, at otherwise the same parameters as specified 


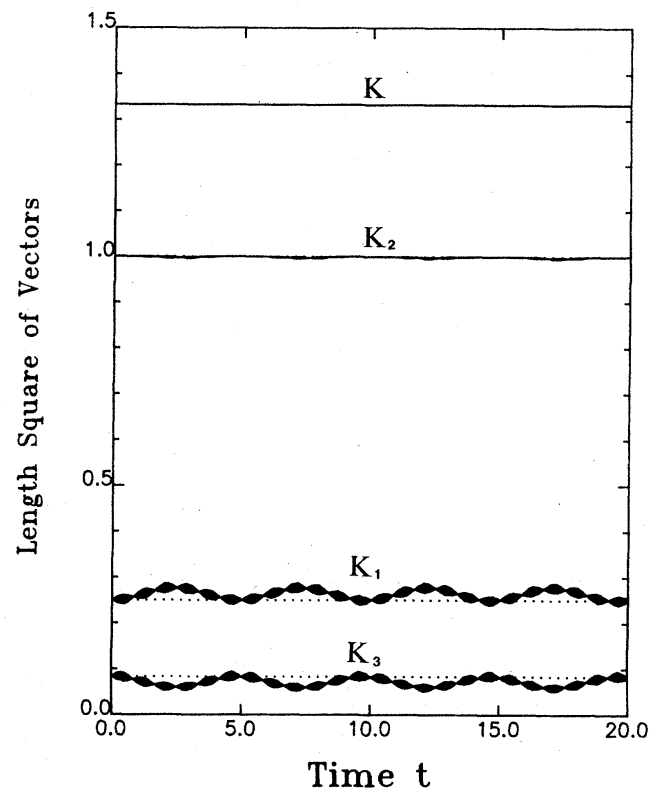

FIG. 9. The length squares of the subvectors (a) $K_{1}=|\mathbf{A}(t)|^{2}$, (b) $K_{2}=|\mathbf{B}(t)|^{2}$, and (c) $K_{3}=|\mathbf{C}(t)|^{2}$, and of the total coherence vector, $K=|\mathbf{S}(t)|^{2}=\frac{4}{3}$, as functions of time $t$ for the same case as described in Fig. 3. Solid curves, $\operatorname{GVV}(3,1)$ results and dotted curves, RWA results.

in case (1) are presented in Figs. 2(a) and 2(b), respectively. The dispersivelike line shape $\bar{P}_{12}$ beneath the grand $\bar{P}_{13}$ absorption peak around $\Delta_{2}=-5.0$ is similar to the one discussed in paper II and has been discussed thoroughly by $\mathrm{Chu}$ et al. ${ }^{24}$ The time-dependent transition probabilities $P_{11}(t), P_{12}(t)$, and $P_{13}(t)$ are shown in Figs. 3(a)-3(c), respectively. The solid curves are $\operatorname{GVV}(3,1)$, which are not distinguishable from the exact ones (not shown) graphically, while the dotted curves are RWAs. It is seen in this case that the RWA results do resemble $\operatorname{GVV}(3,1)$ 's closely except for some small details due to the presence of antirotating terms. We remark here that we have deliberately made only the antirotating effect visible by choosing large disparities between the two transition frequencies $\left(E_{2}-E_{1}\right)$ and $\left(E_{3}-E_{2}\right)$. The periods characterizing the population oscillations between levels 1 and 3 , and levels 1 and 2 are, respectively, defined by the inverses of the quasienergy differences between each corresponding level, i.e., $T_{13}=2 \pi /\left|\lambda_{3,-1,-1}-\lambda_{100}\right|$, and $T_{12}=2 \pi /$ $\left|\lambda_{2,-1,0}-\lambda_{100}\right|$. Explicitly, in the $\operatorname{GVV}(3,1)$ calculations, $T_{13}=4.8866$ and $T_{12}=0.8362$, while in the RWA's, $T_{13}^{(\mathrm{RWA})}=4 \pi /(p-\Delta)=4.9283 \quad$ and $\quad T_{12}^{(\mathrm{RWA})}=2 \pi / p$ $=0.8322$.

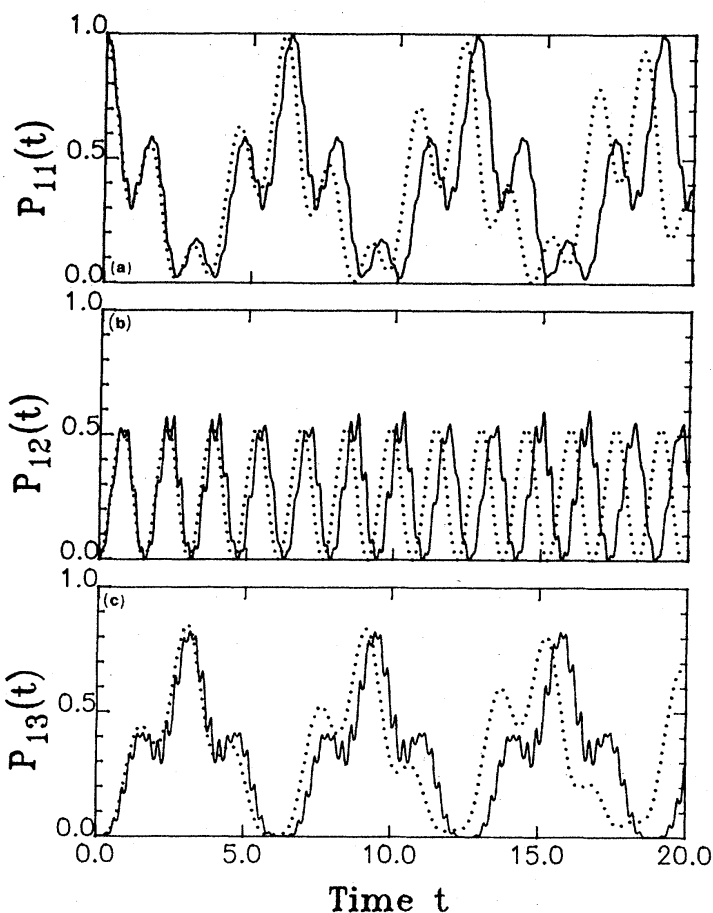

FIG. 10. Time-dependent transition probabilities (a) $P_{11}(t)$, (b) $P_{12}(t)$, and (c) $P_{13}(t)$ for the type-II cascade three-level system at $\left(E_{2}-E_{1}\right)=4000.0,\left(E_{3}-E_{2}\right)=4020.0, \Delta_{1}=-\Delta_{2}=2.0$, $\Omega_{1}=\Omega_{1}^{\prime}=1.5$, and $\Omega_{2}=\Omega_{2}^{\prime}=1.0$. The RWA results, dotted curves, are characterized by periods $T_{12}^{(\mathrm{RWA})}=1.5239$ and $T_{13}^{(\mathrm{RWA})}=5.9189$, while the $\mathrm{GVV}(3,1)$ results, solid curves, are characterized by periods $T_{12}=1.5788$ and $T_{13}=6.1264$. Duration of the first three two-photon flopping periods is shown in this case. For more details see captions in Fig. 3.

Figures 4 and 5 depict the time dependence of the subvectors $\mathbf{A}(t)$ and $\mathbf{B}(t)$ defined in Eqs. (47) and (48). GVV $(3,1)$ results, solid curves, are obtained from Eqs. (33), (58), and (59), while RWA's, dotted curves, from Eqs. (50) and (51). It is seen that components of $\mathbf{A}(t)$ oscillate sinusoidally with the period $T_{12}$, cf. Eqs. (50), while components of $\mathbf{B}(t)$ behave in a slightly more complicated fashion, defined by periods $T_{13}$ and $T_{12}$ together, cf. Eqs. (51). Equations (53) indicate components $A_{1}-A_{2}$, $A_{1}-A_{3}, B_{1}-B_{4}$, and $B_{2}-B_{3}$ are correlated closely in pairs; and Figs. 6(a), 7(a), and $8(a)$, the $\operatorname{GVV}(3,1)$ results, and Figs. 6(b), 7(b), and 8(b), the RWA's, demonstrate these facts. The projection of the RWA trajectory of the coherent vector $\mathrm{S}$ on the $A_{1}-A_{2}$ plane is a perfect ellipse [see Eq. (53a) or Fig. 6(b)], while the counterpart of the $\operatorname{GVV}(3,1)$ 's show some small fluctuation around the el-

TABLE II. Comparison of the quasienergy calculations for the cascade three-level system [type II, Fig. 1(b)] at exact two-photon resonance condition. Parameters used are $E_{1}=0, E_{2}-E_{1}=4000.0$, $E_{3}-E_{2}=4020.0, \Delta_{1}=-\Delta_{2}=2.0, \Omega_{1}=\Omega_{1}^{\prime}=1.5$, and $\Omega_{2}=\Omega_{2}^{\prime}=1.0$ (arbitrary units).

\begin{tabular}{lcccc}
\hline \hline & Exact $[2,2]$ & Exact $[1,1]$ & GVV(3,1) & RWA \\
\hline$\lambda_{100}$ & -1.0022 & -1.0022 & -1.0018 & -1.0616 \\
$\lambda_{2,-1,0}$ & 2.9783 & 2.9783 & 2.9779 & 3.0616 \\
$\lambda_{3,-1,-1}$ & 0.0238 & 0.0238 & 0.0238 & 0.0 \\
\hline \hline
\end{tabular}




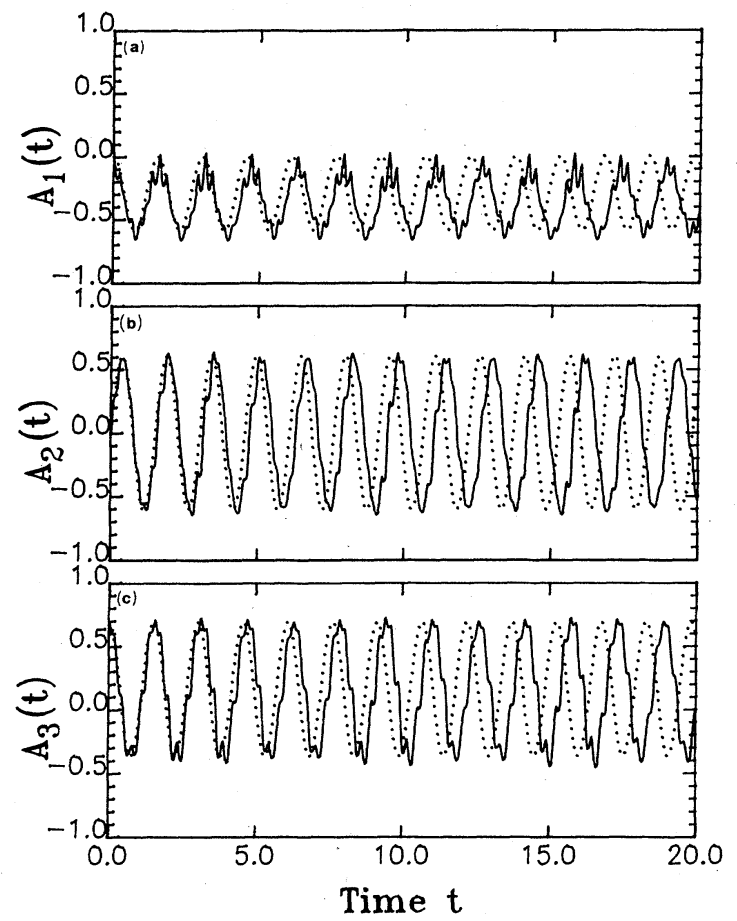

FIG. 11. Components of the subvector $\mathbf{A}(t)$, i.e., (a) $A_{1}(t)$, (b) $A_{2}(t)$, and (c) $A_{3}(t)$, as functions of time $t$. Parameters same as Fig. 10.

lipse, Fig. 6(a). This small deviation is mainly caused by the antirotating terms. The RWA projection on the $B_{1}$ $B_{4}$ plane, Fig. $7(\mathrm{~b})$, is described by Eq. (53d). It swings with an angular frequency $p=2 \pi / T_{12}^{(\mathrm{RWA})}$ and an ampli-
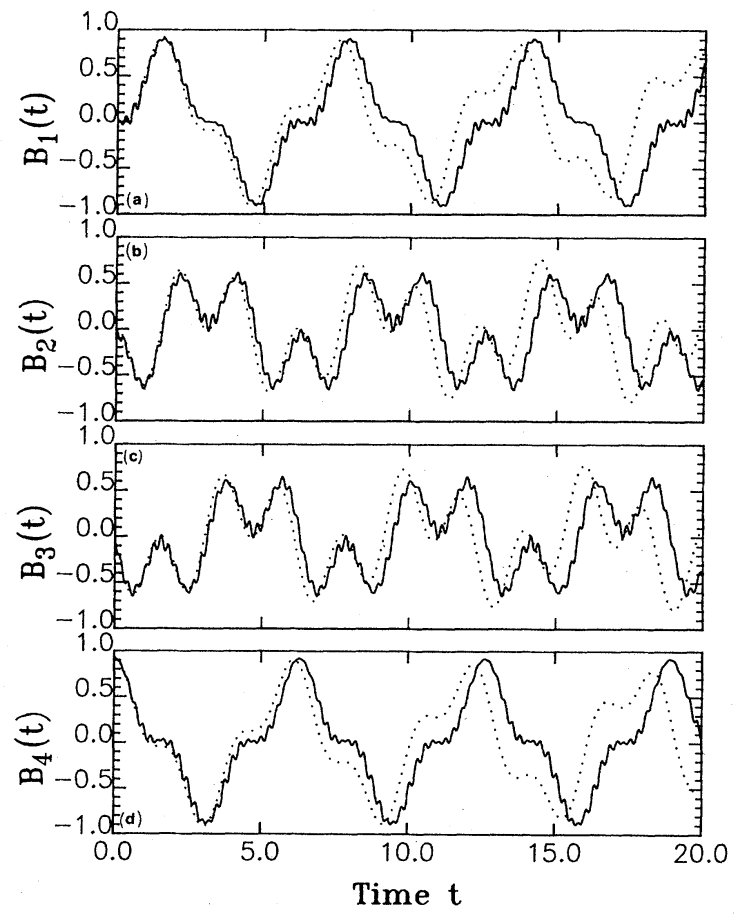

FIG. 12. Components of the subvector $\mathbf{B}(t)$, i.e., (a) $B_{1}(t)$, (b) $B_{2}(t)$, and (c) $B_{3}(t)$, and (d) $B_{4}(t)$, as functions of time $t$. $\mathrm{Pa}$ rameters same as Fig. 10.

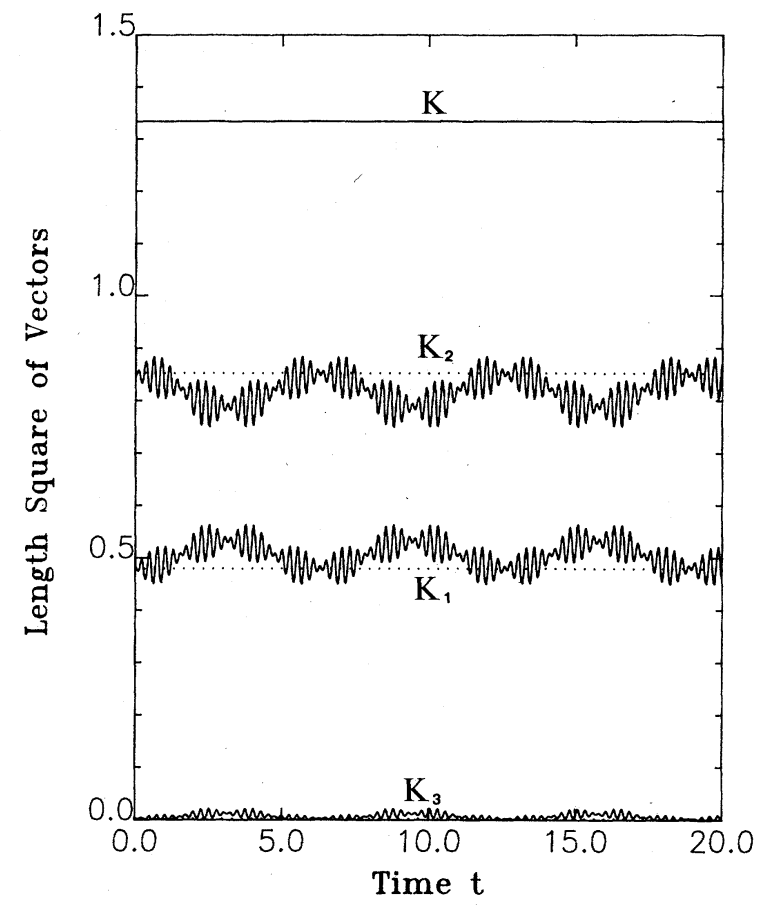

FIG. 13. The length squares of the subvectors (a) $K_{1}=|\mathbf{A}(t)|^{2}$, (b) $K_{2}=|\mathbf{B}(t)|^{2}$, and (c) $K_{3}=|\mathbf{C}(t)|^{2}$, and of the total coherence vector $K=|\mathbf{S}(t)|^{2}=\frac{4}{3}$ as functions of time $t$ for the same case as described in Fig. 10. Solid curves, $\operatorname{GVV}(3,1)$ results and dotted curves, RWA results.

tude $4 \Omega_{1}^{2}\left(\Omega_{2}^{\prime}\right)^{2}\left(p^{2}-4 b^{2}\right) /\left(b^{4} p^{2}\right)$ while circulating in a frequency $2 \pi / T\left(T_{3}^{\text {RWA })}\right.$ and a radius $K_{2}=4 \Omega_{1}^{2}\left(\Omega_{2}^{\prime}\right)^{2} / b^{4}$. The GVV $(3,1)$ counterpart on the $B_{1}-B_{4}$ plane, Fig. $7(a)$, shows deviations, from the RWA's, due to the antirotating terms: the frequencies of the swinging and the circulation have been slightly increased, and small wiggling structures show off. The RWA projection on the $B_{2}-B_{3}$ plane, Fig. 8(b), can be understood from Eq. (53c). It swings and circulates in the same amplitude $8 \Omega_{1}^{2}\left(\Omega_{2}^{\prime}\right)^{2} /\left(b^{2} p^{2}\right)$, but with different frequencies, $p$ and $2 \pi / T_{13}^{(\mathrm{RWA})}$, respectively. The $\operatorname{GVV}(3,1)$ counterpart on the $B_{2}-B_{3}$ plane is shown in Fig. 8(a). In Fig. 9 we sketch the lengths of the three subvectors $\mathbf{A}(t), \mathbf{B}(t)$, and $\mathbf{C}(t)$, and of the coherence vector $\mathbf{S}(t)$ as a function of time. The dotted lines show constancies of the subvectors in the RWA limit, while the solid curves show the corresponding $\operatorname{GVV}(3,1)$ symmetry-breaking results. In this case the coupling between the vectors $\mathbf{A}(t)$ and $\mathbf{C}(t)$ appears much stronger than their individual coupling with the vector $\mathbf{B}(t)$, though the length of the vector $\mathbf{B}(t)$ also exhibits small oscillations away from the RWA constant. The length of the coherence vector $\mathbf{S}(t)$ stays constant in either calculation and assumes the value $K=|\mathbf{S}(t)|^{2}=\frac{4}{3}$, as it should be.

\section{B. Type-II three-level system at exact two-photon resonance}

We assume that $\left(E_{2}-E_{1}\right)=4000.0,\left(E_{3}-F_{2}\right)=4020.0$, $\Delta_{1}=-\Delta_{2}=\Delta=2.0, \Omega_{1}=\Omega_{1}^{\prime}=1.5$, and $\Omega_{2}=\Omega_{2}^{\prime}=1.0$. The 
TABLE III. Comparison of the exact quasienergy calculations for the cascade three-level system [type II, Fig. 1(b)] at nearly two-photon resonance condition. Parameters used are $E_{1}=0$, $E_{2}-E_{1}=4000.0, E_{3}-E_{2}=4020.0, \Delta_{1}=2.0, \Delta_{2}=-1.8$, and $\Omega_{1}=\Omega_{1}^{\prime}=\Omega_{2}=\Omega_{2}^{\prime}=1.0$ (arbitrary units).

\begin{tabular}{lccrr}
\hline \hline & Exact [2,2] & Exact [1,1] & GVV(3,1) & RWA \\
\hline$\lambda_{100}$ & -0.6103 & -0.6104 & -0.6102 & -0.6639 \\
$\lambda_{2,-1,0}$ & 2.7006 & 2.7006 & 2.7004 & 2.7545 \\
$\lambda_{3,-1,-1}$ & 0.1098 & 0.1098 & 0.1098 & 0.1094 \\
\hline \hline
\end{tabular}

small difference between the two allowed transition frequencies $\left(E_{2}-E_{1}\right)$ and $\left(E_{3}-E_{2}\right)$ implies that the only significant non-RWA effects is from the possible competition of the transition via the non-RWA channel, while the effect due to the antirotating pairs is totally negligible. We note that the RWA channel represents two sequential near-resonant one-photon absorptions,

$$
|1\rangle \stackrel{\omega_{1}}{\rightarrow}|2\rangle \stackrel{\omega_{2}}{\rightarrow}|3\rangle
$$

where the non-RWA channel comprises two nonresonant one-photon absorptions,

$$
|1\rangle \stackrel{\omega_{2}}{\rightarrow}|2\rangle \stackrel{\omega_{1}}{\rightarrow}|3\rangle
$$
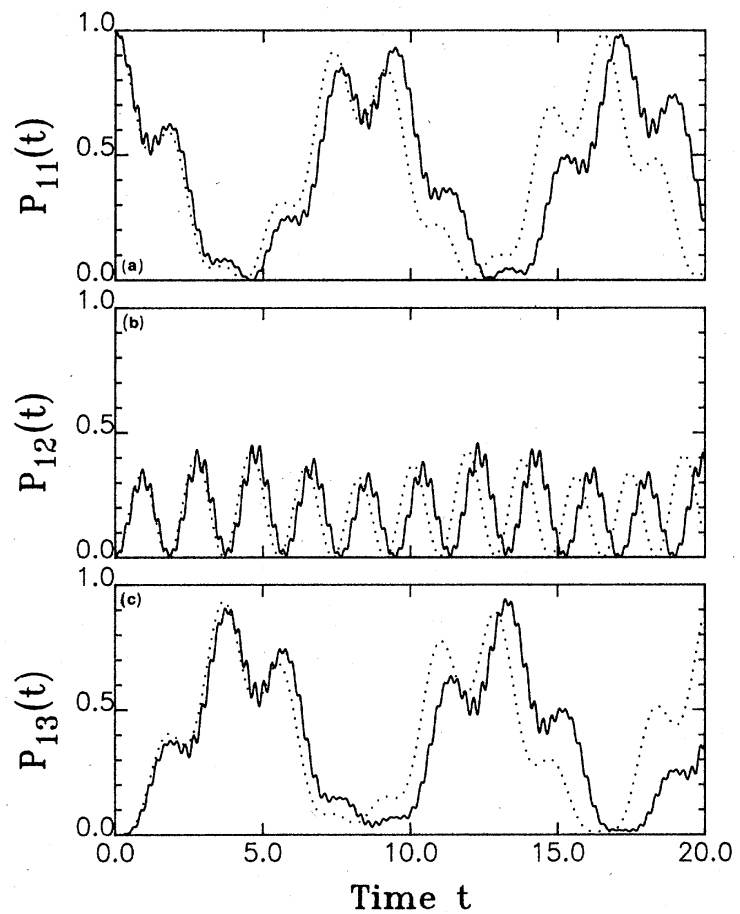

FIG. 14. Time-dependent transition probabilities (a) $P_{11}(t)$, (b) $P_{12}(t)$, and (c) $P_{13}(t)$ for the type-II cascade three-level system at $\left(E_{2}-E_{1}\right)=4000.0, \quad\left(E_{3}-E_{2}\right)=4020.0, \quad \Delta_{1}=2.0$, $\Delta_{2}=-1.8$, and $\Omega_{1}=\Omega_{1}^{\prime}=\Omega_{2}=\Omega_{2}^{\prime}=1.0$. The RWA results, dotted curves, are characterized by periods $T_{12}^{(\mathrm{RWA})}=1.8381$ and $T_{13}^{(\mathrm{RWA})}=8.1257$, while the $\operatorname{GVV}(3,1)$ results, solid curves, are characterized by periods $T_{12}=1.8977$ and $T_{13}=8.7254$. Duration of the first two two-photon flopping periods is shown in this case. For more details see captions in Fig. 3. although the two-photon resonance condition is satisfied in either way. We give quasienergies $\lambda_{100}, \lambda_{2,-1,0}$, and $\lambda_{3,-1,-1}$ in Table II which shows again the exact results converge at the photon block [2,2], and $\operatorname{GVV}(3,1)$ 's are still in very good agreement to the exact ones. But the RWA results are not as close to the exact ones as in the case (1), cf. Eqs. (35)-(37). In Figs. 10 we present the time-dependent transition probabilities $P_{11}(t), P_{12}(t)$, and $P_{13}(t)$ of the $\operatorname{GVV}(3,1)$, solid curves, and of the RWA, dotted curves, results. In this case we observe that not only the characteristic periods $T_{13}=6.1264$ and $T_{12}=1.5788$ for the $\operatorname{GVV}(3,1)$ and $T_{13}^{(\mathrm{RWA})}=5.9189$ and $T\left({ }_{12}^{(\mathrm{WA})}=1.5239\right.$ for the RWA are quite different, but also their details in Rabi flopping patterns. While the RWA curves are always smooth, the non-RWA transition channels introduce further oscillations riding on the RWA-like profiles. The amplitude of these fluctuations is proportional to the quantities $\left|\Omega_{2} /\left[\left(\omega_{2}-\omega_{1}\right)-\Delta_{1}\right]\right|$ and $\left|\Omega_{1}^{\prime} /\left[\left(\omega_{2}-\omega_{1}\right)+\Delta_{2}\right]\right|$, i.e., Eqs. (36) and (37).

The time-dependent components of the subvectors $\mathbf{A}(t)$

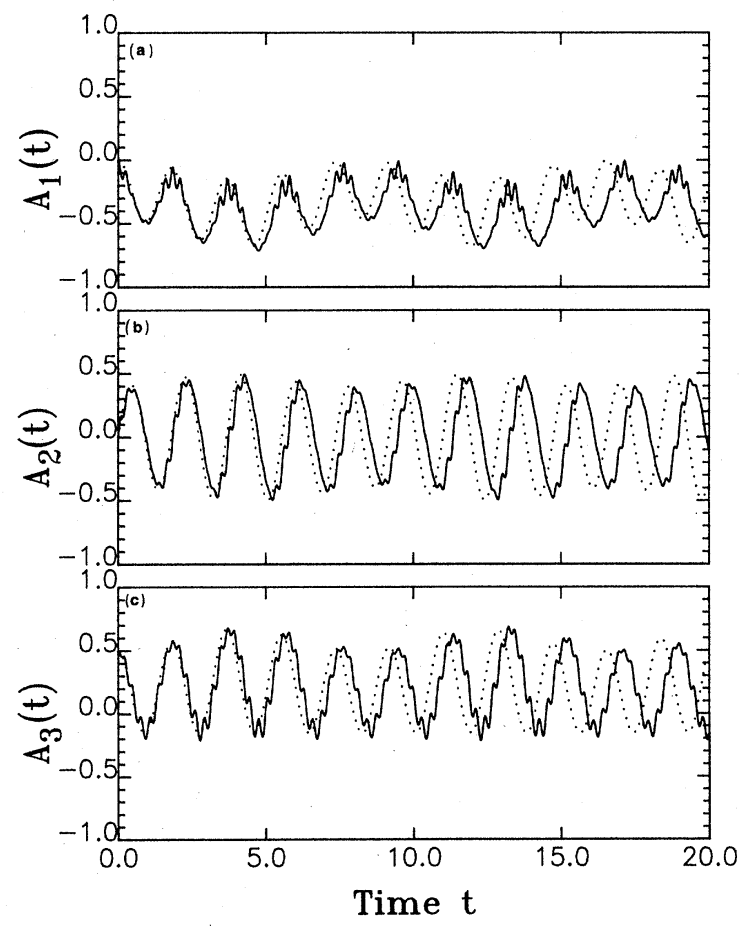

FIG. 15. Components of the subvector $\mathbf{A}(t)$, i.e., (a) $A_{1}(t)$, (b) $A_{2}(t)$, and (c) $A_{3}(t)$, as functions of time $t$ for the same case and time duration as described in Fig. 14. 


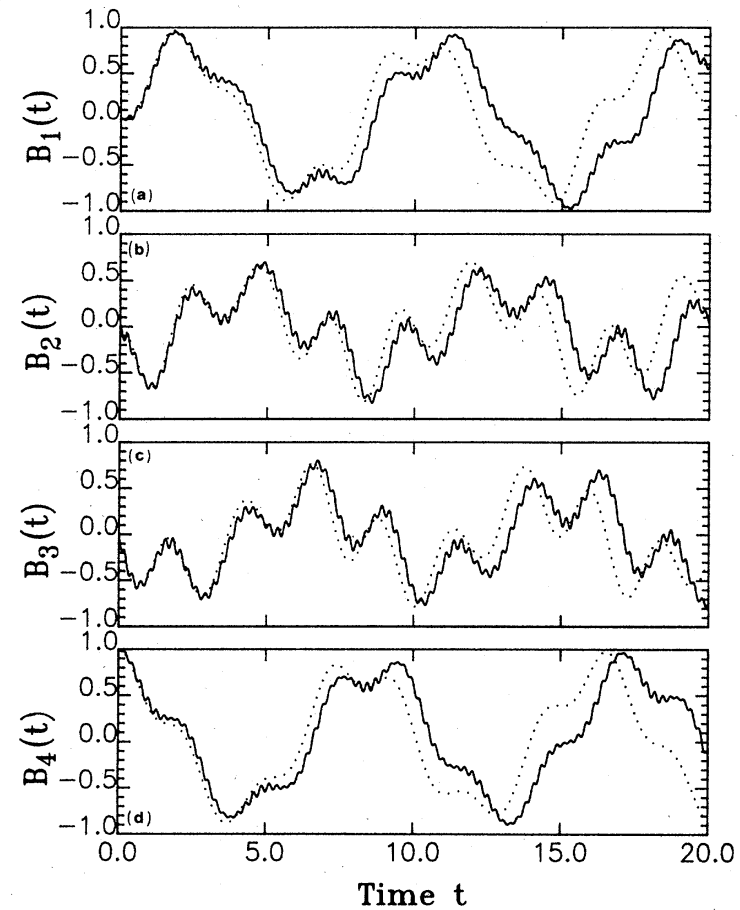

FIG. 16. Components of the subvector $\mathbf{B}(t)$, i.e., (a) $B_{1}(t)$, (b) $B_{2}(t)$, (c) $B_{3}(t)$, and (d) $B_{4}(t)$, as functions of time $t$ for the same case and time duration as described in Fig. 14.
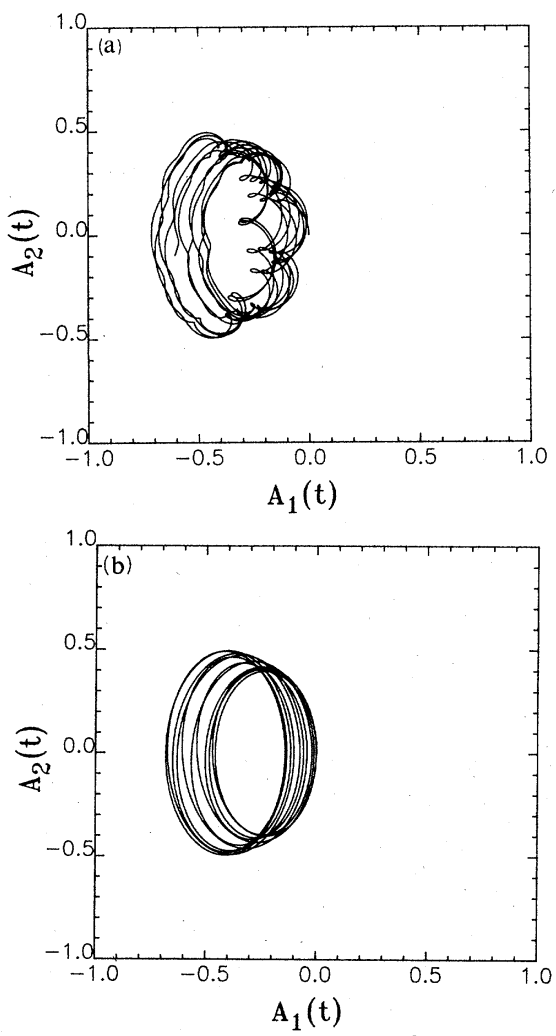

FIG. 17. Projection of the trajectory of the subvector $\mathbf{A}(t)$ on the $A_{1}-A_{2}$ plane for the same case and time duration as shown in Fig. 15. (a) $\operatorname{GVV}(3,1)$ results and (b) RWA results.
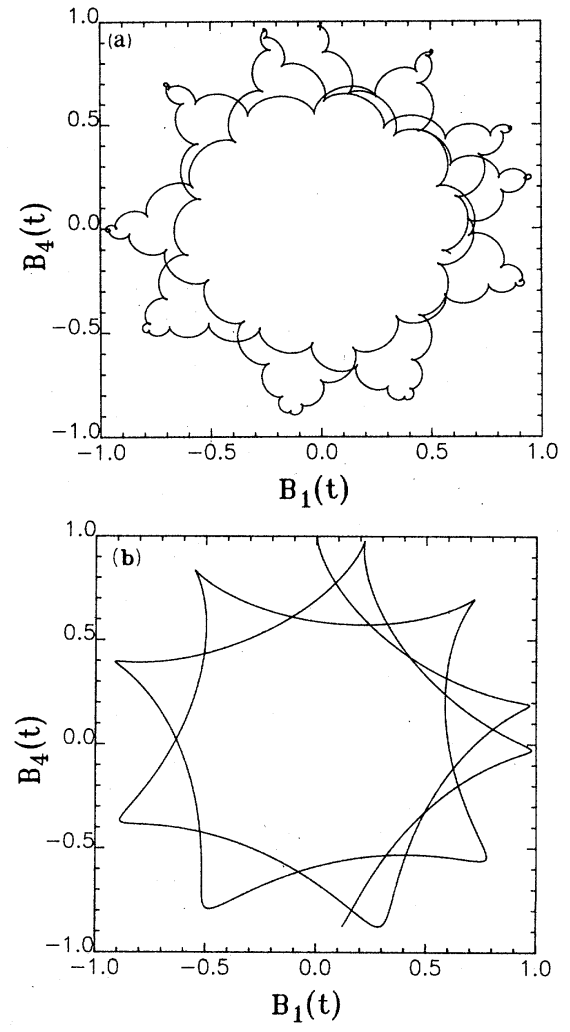

FIG. 18. Projection of the trajectory of the subvector $\mathbf{B}(t)$ on the $B_{1}-B_{4}$ plane for the same case and time duration as shown in Fig. 16. (a) GVV $(3,1)$ results and (b) RWA results.

and $\mathbf{B}(t)$ are shown in Figs. 11 and 12. The projections of the coherence vector $S(t)$, of the $\operatorname{GVV}(3,1)$ calculation, on the $A_{1}-A_{2}, B_{1}-B_{4}$, and $B_{2}-B_{3}$ plane exhibit roughly similar geometrical patterns as shown in Figs. 6(a), 7(a), and 8(a). However, the deviations of these projections from the corresponding RWA patterns are rather large in this case (not shown). These differences are also manifested in the time dependence of the lengths of the three subvectors $\mathbf{A}(t), \mathbf{B}(t)$, and $\mathbf{C}(t)$, see Fig. 13. In this case it is found that the coupling between $\mathbf{A}(t)$ and $\mathbf{B}(t)$ is stronger than that between either of $\mathbf{A}(t)$ and $\mathbf{B}(t)$ with $\mathbf{C}(t)$. Nevertheless the length of the $\mathbf{S}(t)$, i.e., $K$, remains constant at $\frac{4}{3}$. We should remark that had we chosen $\Omega_{1} \Omega_{1}^{\prime}=\Omega_{2} \Omega_{2}^{\prime}$, the couplings amongst the subvectors $\mathbf{A}(t)$, $\mathbf{B}(t)$, and $\mathbf{C}(t)$ would have been approximately zero in this case, although the subvectors $\mathbf{A}(t)$ and $\mathbf{B}(t)$ would behave quite differently from what the RWA has projected, cf. Eqs. (57).

\section{Type-II three-level system at nearly two-photon resonance}

In this case we set $\left(E_{2}-E_{1}\right)=4000.0,\left(E_{3}-E_{2}\right)$ $=4020.0, \quad \Delta_{1}=2.0, \quad \Delta_{2}=-1.8, \quad \Omega_{1}=\Omega_{1}^{\prime}=1.0, \quad$ and $\Omega_{2}=\Omega_{2}^{\prime}=1.0$. Here we have purposely made the products $\Omega_{1} \Omega_{1}^{\prime}$ and $\Omega_{2} \Omega_{2}^{\prime}$ equal so that couplings amongst $\mathbf{A}(t)$, $\mathbf{B}(t)$, and $\mathbf{C}(t)$ and the symmetry-breaking effects are predominantly caused by the unequal detunings 

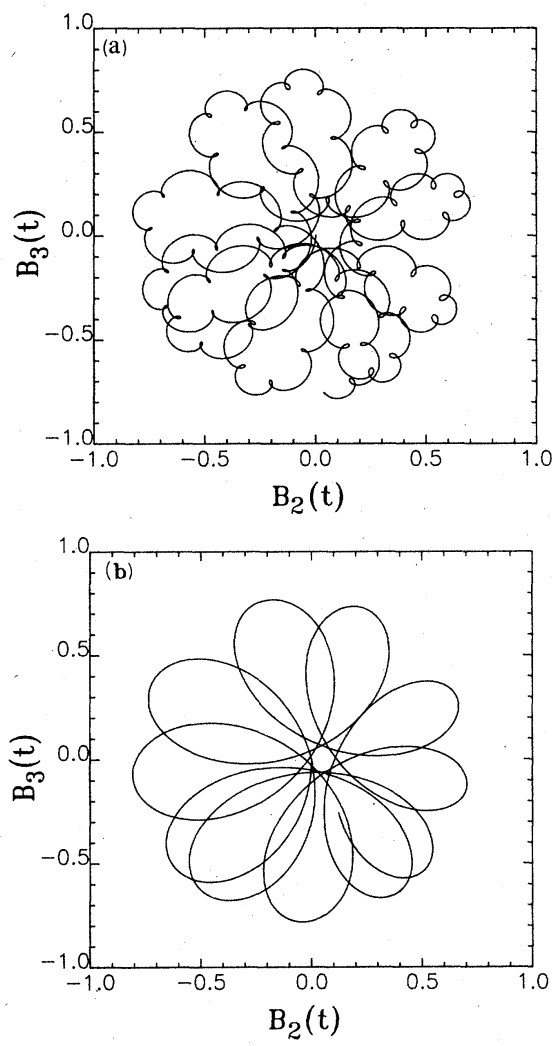

FIG. 19. Projection of the trajectory of the subvector $\mathbf{B}(t)$ on the $B_{2}-B_{3}$ plane for the same case and time duration as shown in Fig. 16. (a) GVV $(3,1)$ results and (b) RWA results.

$\Delta_{1} \neq-\Delta_{2}$, or $\Delta_{1}+\Delta_{2} \neq 0$. Table III reports the quasienergies, for this case, of the exact $\operatorname{GVV}(3,1)$ and the RWA calculations, respectively. The $\operatorname{GVV}(3,1)$ 's do agree with the converged exact [2,3] calculations, while the RWA's are a little bit off the exact ones. Figures 14 show the GVV $(3,1)$ results, solid curves, and the RWA's, dotted curves, for the time-dependent transition probabilities $P_{11}(t), P_{12}(t)$, and $P_{13}(t)$. The characteristic periods for the former are $T_{13}=8.7254$ and $T_{12}=1.8977$, while for the latter $T_{13}^{(\mathrm{RWA})}=8.1257$ and $T_{12}^{(\mathrm{RWA})}=1.8381$. The small fluctuation structures riding on the $\operatorname{GVV}(3,1)$ profiles are due to the non-RWA transition channels which still play roles within each subvector $\mathbf{A}(t)$ and $\mathbf{B}(t)$.

In Figs. 15 and 16 we present the components of the subvectors $\mathbf{A}(t)$ and $\mathbf{B}(t)$ as functions of time. The RWA projection of the $S(t)$ on the $A_{1}-A_{2}$ plane, Fig. 17(b), shows that the two-photon resonance ellipse, e.g., Fig. 6(b), can no longer hold. It advances back and forth in varying radius, although it is still very much confined to a small area. The $\operatorname{GVV}(3,1)$ correspondence of this illustrates the complication caused by the non-RWA transition channels. Figures $18(\mathrm{~b})$ and 19(b) are projections of the RWA coherence vectors on the $B_{1}-B_{4}$ and the $B_{2}-B_{3}$ planes. The more symmetrical patterns of their twophoton resonance counterparts, e.g., Figs. 7(b) and 8(b), are somehow distorted in the case of non-two-photon resonance. Figures 18(a) and 19(a) depict the same projections from the GVV $(3,1)$ calculations but exhibit very dif-

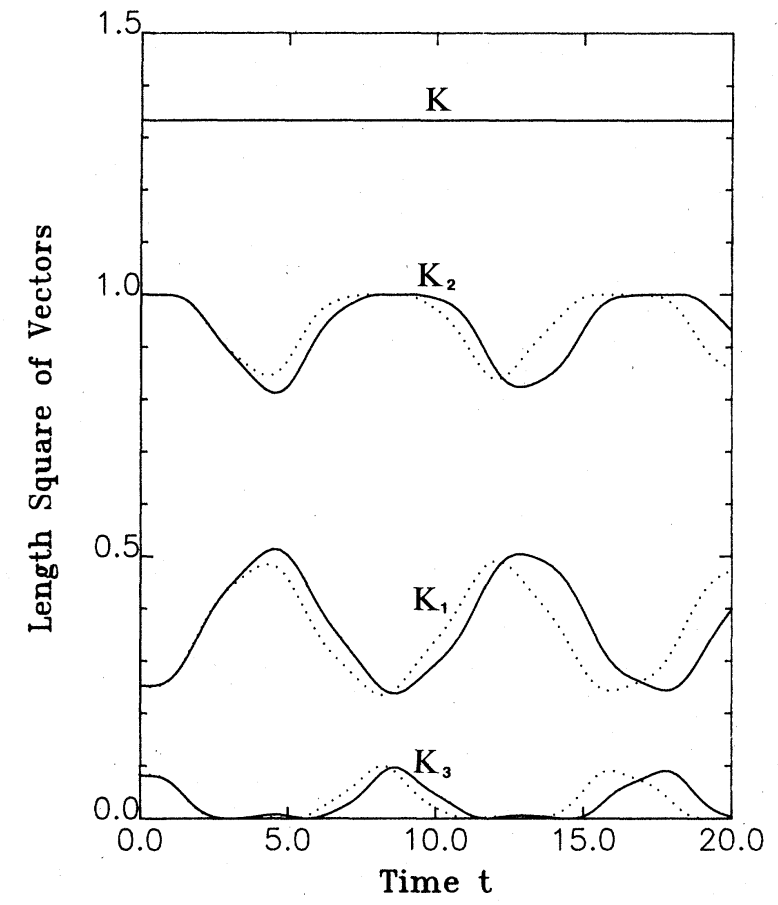

FIG. 20. The length squares of the subvectors (a) $K_{1}=|\mathbf{A}(t)|^{2}$, (b) $K_{2}=|\mathbf{B}(t)|^{2}$, and (c) $K_{3}=|\mathbf{C}(t)|^{2}$, and of the total coherence vector, $K=|\mathbf{S}(t)|^{2}=\frac{4}{3}$, as functions of time $t$ for the same case as described in Fig. 14. Solid curves, GVV $(3,1)$ results and dotted curves, RWA results.

ferent geometrical patterns. Finally, in Fig. 20 we show the dependence of the length of each of the subvectors $\mathbf{A}(t), \mathbf{B}(t)$, and $\mathbf{C}(t)$. It is apparent that the couplings amongst them, because of the nonvanishing quantity $\Delta_{1}+\Delta_{2}$, are quite strong. Equations (55)-(57) indicate that these three subvectors are coupled mainly via the factor $\Gamma_{7}$ of Eq. (57). It is also interesting to see that the small fluctuation structures appearing in Figs. 14-19 do not show up at all even on the $\operatorname{GVV}(3,1)$ calculations in Fig. 20. This can be attributed to the fact that the coupling coefficient $\Gamma_{7}$ is time independent.

\section{CONCLUSION}

We have shown in this paper that the study of the SU(3) dynamical evolution as well as symmetry-breaking phenomena in three-levels systems driven by strong bichromatic fields can be greatly facilitated by the use of the MMFT and expressed in terms of a few timeindependent quasienergy eigenvalues and eigenvectors. Furthermore, we have performed an analytical treatment of the two-mode Floquet Hamiltonian by extending the GVV nearly degenerate perturbation theory. This reduces the infinite-dimensional time-independent Floquet Hamiltonian into a $3 \times 3$ effective Hamiltonian, from which analytical properties of the SU(3) coherent vector can be readily obtained. The combination of the MMFT and the GVV method thus yield a practical and powerful nonper- 
turbative analytical technique for the exploration of the dynamical evolution. The method is applied to the first detailed pictorial study of the time evolution of the eightdimensional SU(3) coherent vector in cascade three-level systems near two-photon resonance induced by two strong, linearly polarized monochromatic fields.

We are currently extending the method to incorporate the effects of damping mechanisms as well as multiphoton resonances. Results will be published elsewhere.

\section{ACKNOWLEDGMENTS}

This work was supported in part by the U.S. Department of Energy (Division of Chemical Sciences), by the Alfred P. Sloan Foundation (for S.-I C.), and by the University of Kansas (through the General Research Fund). Acknowledgment is also made to the Donors of the Petroleum Research Fund, administered by the American Chemical Society, for partial support of this work.
${ }^{1}$ Y. Zur and S. Vega, J. Chem. Phys. 79, 548 (1983), and references therein.

${ }^{2}$ K. Shimoda, Laser Spectroscopy of Atoms and Molecules, edited by H. Walther (Springer, Berlin, 1976), pp. 197-252; H. W. Galbrait, M. Dubs, and J. I. Steinfeld, Phys. Rev. A 26, 1528 (1982), and references therein.

${ }^{3}$ M. Takatsuji, Phys. Rev. A 4, 808 (1971); D. Grischkowsky, M. M. T. Loy, and P. F. Liao, ibid. 12, 2514 (1975), and references therein.

${ }^{4}$ R. G. Brewer and E. L. Hahn, Phys. Rev. A 11, 1641 (1975).

${ }^{5}$ See, for example, P. M. Radmore and P. L. Knight, J. Phys. B 15, 561 (1982); M. Kaivola, N. Bjerre, O. Poulsen, and J. Javanainen, Opt. Commun. 49, 418 (1984); L. Kancheva, D. Pushkarov, and S. Rashev, J. Phys. B 14, 573 (1981); D. T. Pegg, ibid. 16, 2135 (1983).

${ }^{6}$ C. Cohn-Tannoudji and S. Reynaud, J. Phys. B 10, 365 (1977); G. S. Agarwa and S. S. Jha, ibid. 12, 2655 (1979).

${ }^{7}$ R. P. Feynman, F. L. Vernon, Jr., and R. W. Hellwarth, J. Appl. Phys. 28, 49 (1957).

${ }^{8}$ L. Allen and J. H. Eberly, Optical Resonance and Two-Level Atoms, (Wiley, New York, 1975), Chap. 2.

${ }^{9}$ F. T. Hioe and J. H. Eberly, Phys. Rev. Lett. 47, 838 (1981).

10J. N. Elgin, Phys. Lett. 80A, 140 (1980).

${ }^{11}$ S. Vega and A. Pines, J. Chem. Phys. 66, 5624 (1977); S. Vega, ibid. 68, 5518 (1978).

${ }^{12}$ F. T. Hioe and J. H. Eberly, Phys. Rev. A 25, 2168 (1982); F. T. Hioe, ibid. 28, 879 (1983); F. T. Hioe and J. H. Eberly, ibid. 29, 1164 (1984).

13J. Oreg, F. T. Hioe, and J. H. Eberly, Phys. Rev. A 29, 690
(1984).

${ }^{14}$ See, for example, S. I. Chu, J. V. Tietz, and K. K. Datta, J. Chem. Phys. 77, 2968 (1982); K. F. Milfeld and R. E. Wyatt, Phys. Rev. A 27, 72 (1983); J. V. Tietz and S. I. Chu, Chem. Phys. Lett. 101, 446 (1983); T. S. Ho and S. I. Chu, J. Chem. Phys. 79, 4708 (1983).

${ }^{15}$ S. I. Chu and W. P. Reinhardt, Phys. Rev. Lett. 39, 1195 (1977); S. I. Chu, Chem. Phys. Lett. 54, 367 (1978); A. Maquet, S. I. Chu, and W. P. Reinhardt, Phys. Rev. A 27, 2946 (1983).

${ }^{16}$ See, for example, S. I. Chu, J. Chem. Phys. 75, 2215 (1981); S. I. Chu, C. Laughlin, and K. K. Datta, Chem. Phys. Lett. 98, 476 (1983); C. Leforestier and R. E. Wyatt, Phys. Rev. A 25, 1250 (1982).

${ }^{17}$ T. S. Ho, S. I. Chu, and J. V. Tietz, Chem. Phys. Lett. 99, 422 (1983).

${ }^{18}$ T. S. Ho and S. I. Chu, J. Phys. B 17, 2101 (1984).

${ }^{19}$ J. H. Shirley, Phys. Rev. 138, B979 (1965).

${ }^{20}$ H. Salwen, Phys. Rev. 99, 1274 (1955).

${ }^{21}$ B. Kirtman, J. Chem. Phys. 49, 3890 (1968); 75, 798 (1981).

22P. R. Certain and J. O. Hirschfelder, J. Chem. Phys. 52, 5977 (1970).

23J. H. Van Vleck, Phys. Rev. 33, 467 (1929).

${ }^{24}$ P. K. Aravind and J. O. Hirschfelder, J. Phys. Chem. 88, 4788 (1984).

${ }^{25}$ S. I. Chu, J. V. Tietz, and K. K. Datta, J. Chem. Phys. 77, 2968 (1983).

${ }^{26} \mathrm{M}$. Gell-Man and Y. Néeman, The Eight-Fold Way (Benjamin, New York, 1964). 